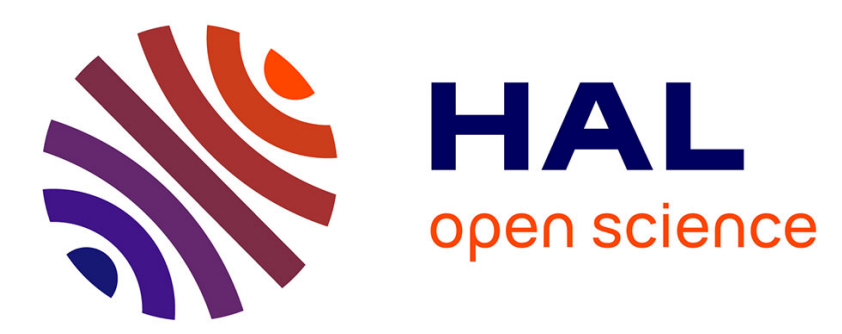

\title{
Front-tracking by the level-set and the volume penalization methods in a two-phase microfluidic network
}

\author{
Johana Pinilla, Charles-Henri Bruneau, Sandra Tancogne
}

\section{To cite this version:}

Johana Pinilla, Charles-Henri Bruneau, Sandra Tancogne. Front-tracking by the level-set and the volume penalization methods in a two-phase microfluidic network. International Journal for Numerical Methods in Fluids, 2016, 80 (1), pp.23-52. 10.1002/fld.4069 . hal-01251457

\author{
HAL Id: hal-01251457 \\ https://hal.inria.fr/hal-01251457
}

Submitted on 6 Jan 2016

HAL is a multi-disciplinary open access archive for the deposit and dissemination of scientific research documents, whether they are published or not. The documents may come from teaching and research institutions in France or abroad, or from public or private research centers.
L'archive ouverte pluridisciplinaire HAL, est destinée au dépôt et à la diffusion de documents scientifiques de niveau recherche, publiés ou non, émanant des établissements d'enseignement et de recherche français ou étrangers, des laboratoires publics ou privés. 


\title{
Front-tracking by the level-set and the volume penalization methods in a two-phase microfluidic network
}

\author{
Johana Pinilla ${ }^{1}$, Charles-Henri Bruneau ${ }^{1}$, Sandra Tancogne $e^{1,2}$ \\ 1 Univ. Bordeaux, IMB. \\ CNRS, IMB, UMR 5251. \\ INRIA Bordeaux, Team MC2. \\ F-33400 Talence, France. \\ 2 Laboratoire de Mathématiques \\ Université de Reims Champagne-Ardenne - B.P. 1039 \\ F-51687 Reims, France.
}

\begin{abstract}
Two-phase immiscible fluids in a two-dimensional micro-channels network are considered. The incompressible Stokes equations are used to describe the Newtonian fluid flow while the Oldroyd-B rheological model is used to capture the viscoelastic behavior. In order to perform numerical simulations in a complex geometry like a micro-channels network, the volume penalization method is implemented. To follow the interface between the two fluids, the level-set method is used and the dynamics of the contact line is modeled by Cox law. Numerical results show the ability of the method to simulate two-phase flows and to follow properly the contact line between the two immiscible fluids. Finally, simulations with realistic parameters are performed to show the difference when a Newtonian fluid is pushed by a viscoelastic fluid instead of a Newtonian one.
\end{abstract}

Keywords: microfluidics, two-phase flow, triple line, contact line, Cox model, volume penalization method, level-set method, polymer, Oldroyd-B equations

\section{Introduction}

Micro-channels networks are frequently used in laboratories to mimic the flow conditions in a porous medium at the micro scale [11]. The main purpose of the researchers is to show that some parts of the micro-channels can be reached by modifying the fluid properties.

At this scale, the flow is generally laminar and the motion of the interface between the two immiscible Newtonian fluids is controlled by the surface tension. The modeling of two-phase flow at micro scale has already been studied in the past (for example [49, 52]) using the incompressible Stokes equations with various viscosities and the level-set method to follow the interfaces. These works allowed 
to study, respectively in three-dimensions and in two-dimensions, the creation of droplets in micro-channels and to bind the experimental data like the flow rates or the viscosities to the shapes and internal dynamics of the microdroplets.

One of the most important aspects to consider in two-phase flow simulation at the micro-scale, is the motion of the contact line. Many attempts to simulate the dynamics of the contact line have been developed, a very good review of these methods can be found in $[36,16,45]$. The dynamics of the contact line used in this work is based on the theoretical Cox analysis [12]. Indeed, Cox provided a general hydrodynamic description of a moving contact line, that links the triple line velocity to the dynamic contact angle.

The aim of this work is to describe a numerical model to simulate in twodimensions a two-phase flow of Newtonian and viscoelastic fluids, like polymeric solutions, into a micro-channels, including a dynamic contact angle procedure that describes the fluid-fluid-wall dynamics. To handle complex geometries, the volume penalization method is used, adding a penalization term to the Stokes equations to force the boundary condition on the solid parts.

This paper is structured as follows. The physical modeling is presented in section 2 with emphasis to the contact line problem. Then the mathematical modeling is carefully detailed in section 3. Section 4 deals with the numerical resolution and the numerical treatment of the triple lines to move properly the contact line. Section 5 is devoted to a validation test. In section 6 , the numerical results in a single channel are discussed. Finally in the last section, the simulations in a micro-channels network are shown.

\section{Physical modeling}

\subsection{Micro-scale modeling}

Incompressible fluid flows are considered and modelized by means of NavierStokes equations:

$$
\left\{\begin{array}{l}
\nabla \cdot \mathbf{U}=0 \\
\rho\left(\frac{\partial \mathbf{U}}{\partial t}+\mathbf{U} \cdot \nabla \mathbf{U}\right)+\nabla p-\nabla \cdot \boldsymbol{T}=0,
\end{array}\right.
$$

where $\mathbf{U}$ is the velocity, $p$ the pressure, $\rho$ the density and $\boldsymbol{T}$ the extra-stress tensor. In microfluidics, the diameter of the channels is of the order of a few tens of micrometers while the velocity is of the order of $1 \mathrm{~cm} / \mathrm{s}$. In addition, the flow quickly reaches a steady state and the viscous effects dominate the inertial ones. Taking into account these assumptions the first two terms from the motion equation of (1) can be neglected. The system (1) becomes the steady Stokes problem:

$$
\left\{\begin{array}{l}
\nabla \cdot \mathbf{U}=0 \\
\nabla \cdot \boldsymbol{T}=\nabla p .
\end{array}\right.
$$

In the case of a Newtonian fluid, the relation between $\boldsymbol{T}$ and the deformation 
tensor $\mathbf{D}$ is linear and the proportionality constant is the fluid viscosity $\eta$. This leads to:

$$
\boldsymbol{T}=2 \eta \mathbf{D}
$$

where $\mathbf{D}$ is given by

$$
\mathbf{D}=\frac{\nabla \mathbf{U}+(\nabla \mathbf{U})^{t}}{2}
$$

Substituting (3) in (2), we have the Stokes equations to model an incompressible and isothermal flow of a Newtonian fluid at microscopic scale:

$$
\left\{\begin{aligned}
\nabla \cdot \mathbf{U} & =0 \\
\nabla p-\eta \Delta \mathbf{U} & =\mathbf{0} .
\end{aligned}\right.
$$

In the case of a non-Newtonian fluid, like a polymeric solution, the relation between $\boldsymbol{T}$ and $\mathbf{D}$ is non-linear. The extra-stress tensor $\boldsymbol{T}$ is decomposed into two parts: a Newtonian contribution denoted $\boldsymbol{T}_{\boldsymbol{s}}$ and a polymeric contribution denoted $\boldsymbol{T}_{\boldsymbol{p}}$, namely

$$
T=T_{s}+T_{p}
$$

The Newtonian contribution can be calculated from (3). Then, the extra-stress tensor $\boldsymbol{T}$ is given by:

$$
\boldsymbol{T}=2 \eta \mathbf{D}+\boldsymbol{T}_{\boldsymbol{p}}
$$

where $\eta$ is the solvent viscosity. Substituting (6) in (2) yields:

$$
\left\{\begin{aligned}
\nabla \cdot \mathbf{U} & =0 \\
\nabla p-\eta \Delta \mathbf{U}-\nabla \cdot \boldsymbol{T}_{\boldsymbol{p}} & =\mathbf{0} .
\end{aligned}\right.
$$

It still remains to define an equation for $\boldsymbol{T}_{\boldsymbol{p}}$. In the literature, many "appropriate" models are proposed for $\boldsymbol{T}_{\boldsymbol{p}}$ (see $[3,27]$ ). In order to choose this model, several factors must be considered, such as the characteristics of the polymer or the polymer concentration [27]. In this work, the Oldroyd-B model is used for modeling the polymer solution. The Oldroyd-B model is a linear viscoelasticity model which is well adapted for dilute polymer solutions [27]. The Oldroyd-B model written in terms of the conformation tensor reads:

$$
\left\{\begin{aligned}
\nabla \cdot \mathbf{U} & =0, \\
\nabla p-\eta \Delta \mathbf{U}-\frac{\eta_{p}}{\lambda} \nabla \cdot \boldsymbol{\tau} & =\mathbf{0}, \\
\frac{\boldsymbol{\tau}-\mathbf{I}}{\lambda}+\frac{\partial \boldsymbol{\tau}}{\partial t}+(\mathbf{U} \cdot \nabla) \boldsymbol{\tau}-(\nabla \mathbf{U}) \boldsymbol{\tau}-\boldsymbol{\tau}(\nabla \mathbf{U})^{t} & =\mathbf{0} .
\end{aligned}\right.
$$

where $\eta_{p}$ is the polymeric contribution to the solution viscosiy, $\lambda$ is the characteristic relaxation time, $\mathbf{I}$ is the identity tensor and $\boldsymbol{\tau}$ is the conformation tensor that is related to the polymeric tensor $\boldsymbol{T}_{\boldsymbol{p}}$ by:

$$
\boldsymbol{T}_{\boldsymbol{p}}=\frac{\eta_{p}}{\lambda}(\boldsymbol{\tau}-\mathbf{I}) .
$$




\subsection{The contact line problem}

The moving contact line problem is still an issue of controversy and debate $[28,36]$. The difficulty stems from the fact that the classical equations of hydrodynamics, coupled with the no-slip boundary condition leads to a multivalued velocity field. For the fluid, the non-slip boundary condition (zero velocity at the wall) must be satisfied but the triple line is driven by a non-zero velocity. When the no-slip boundary condition is maintained on the wall of a channel for instance, the interface cannot move at the wall and so there is a film of fluid between the wall and the entering flow. Consequently the interface moves faster. Various models have been proposed to deal with this singularity $[35,5,36,33,4]$. Some examples of these models are:

- Precursor film model: This model proposes the existence of a precursor film ahead of the triple line [13]. The precursor film replaces the triple line by a very thin layer of fluid, which eliminates the contact between the fluid/fluid interface and the solid wall. This model can be interpreted as a numerical artefact, with a physical sense, to eliminate the triple line [14]. For more details about the numerical simulation of the triple line with this method see $[7,14,29,31,40]$. The main limitation of this model is the high computational cost: the accuracy of the results is improved by reducing the thickness of the precursor film, however, it requires a very fine spatial resolution [20].

- Diffuse interface models: These models treat the interface as a zone of finite thickness through which the fluid properties vary smoothly. These models allow the contact line to move naturally through the diffusive fluxes even if a no-slip condition is imposed. The diffuse interface model most commonly used to treat problems with triple lines is the Cahn-Hilliard model $[9,15,24,26,41,56]$.

- Slip models: Another solution consists to replace the no-slip boundary condition by a slip condition in the vicinity of the contact line [46, 22]. Slip models have the particularity to introduce a parameter known as the slip length. The slip length is defined as the distance to the wall from which the tangential velocity is zero. Among these models there are the Navier slip boundary condition [34, 21], the stress-free boundary condition [36], the prescribed slip profile [50], the Cox model [12] and other models [4].

For this work, we have selected the hydrodynamic model proposed by Cox [12]. The model developed by Cox, was anticipated by both the experimental work of Hoffman [23] and the analytical work of Dussan [17]. Many numerical studies have made use of this model. For example, Afkhami et al. [1] and Dupont et al. [16] developed a numerical contact angle model for VOF methods, Spelt [46] and Sussman [48] proposed a level-set approach for the simulation of spreading droplets, Zahedi et al. [57] proposed a conservative level-set method for contact line dynamics where the contact angle at equilibrium is used to induce a movement of the contact point by diffusion. In [53] Walker et al. used the explicitly 
tracked contact point moving along the solid boundary introduced by Spelt. A technique to provide an accurate computation of the curvature at the wall is developed and the results are compared with the Cox model for two tests cases. Cox model considers two immiscible phases in contact with an homogeneous solid phase along which the contact line will move with a velocity denoted $V_{c o x}$. Let $U$ be the characteristic velocity of the flow when a fluid 1 (with viscosity $\eta_{1}$ ) is displaced by another fluid 2 (with viscosity $\eta_{2}$ ). If the flows are driven by surface tension, the capillary number $C a=\frac{\eta_{1} U}{\gamma} \ll 1$ with $\gamma$ the surface tension coefficient. Cox [12] provides a general hydrodynamic description of a moving contact line, that links the triple line velocity to the dynamic contact angle by

$$
V_{c o x}=\gamma \frac{g\left(\theta_{d}, q\right)-g\left(\theta_{E}, q\right)}{\eta_{2} \operatorname{Ln}\left(\frac{L}{l_{s}}\right)},
$$

where $\theta_{E}$ is the equilibrium contact angle, $\theta_{d}$ is the dynamic contact angle, $L$ is a macroscopic length, $l_{s}$ is the slip length, $q=\frac{\eta_{2}}{\eta_{1}}$ and

$$
g(\theta, q)=\int_{0}^{\theta} \frac{d \theta}{f(\theta, q)}
$$

with

$$
f(\theta, q)=\frac{2 \sin \theta\left(q^{2}\left(\theta^{2}-\sin ^{2} \theta\right)+2 q\left(\theta(\pi-\theta)+\sin ^{2} \theta\right)+\left((\pi-\theta)^{2}-\sin ^{2} \theta\right)\right)}{q\left(\theta^{2}-\sin ^{2} \theta\right)((\pi-\theta)+\cos \theta \sin \theta)+\left((\pi-\theta)^{2}-\sin ^{2} \theta\right)(\theta-\cos \theta \sin \theta)} .
$$

In the particular case of $q \ll 1$,

$$
g(\theta)=\int_{0}^{\theta} \frac{x-\sin x \cos x}{2 \sin x} d x
$$

In addition, if $\theta_{d}<\frac{3 \pi}{4}, g(\theta) \approx \theta^{3} / 9$ the equation (9) can be approximated by

$$
V_{c o x}=\gamma \frac{\theta_{d}^{3}-\theta_{E}^{3}}{\eta_{2} 9 \operatorname{Ln}\left(\frac{L}{l_{s}}\right)} .
$$

\section{Mathematical modeling}

\subsection{The governing equations}

An incompressible and isothermal flow of two immiscible fluids is considered. In the microfluidic context, the viscous effects are predominant compared to the inertial ones. Thus, the Stokes equations are relevant to model the motion of these fluids. One fluid is Newtonian and the other one is viscoelastic, they will be denoted respectively fluid 1 and fluid 2 . We denote $\Omega$ the domain, $\Omega_{1}$ the sub-domain occupied by fluid 1 and $\Omega_{2}$ the sub-domain occupied by fluid 2 . The interface between the two fluids is defined as $\Sigma=\partial \Omega_{1} \cap \partial \Omega_{2}$, where $\partial \Omega_{i}$ is the 
boundary of $\Omega_{i}$ and $i=\{1,2\}$.

The motion of fluid 1 is governed by the incompressible Stokes equations:

$$
\left\{\begin{array}{l}
\nabla \cdot \mathbf{U}_{1}=0, \\
-\nabla p_{1}+\nabla \cdot\left(2 \eta_{1} \mathbf{D}_{1}\right)=\mathbf{0} .
\end{array}\right.
$$

Whereas the motion of the viscoelastic fluid 2 is governed by Oldroyd-B model:

$$
\left\{\begin{array}{l}
\nabla \cdot \mathbf{U}_{2}=0 \\
-\nabla p_{2}+\nabla \cdot\left(2 \eta_{2} \mathbf{D}_{2}\right)+\frac{\eta_{p_{2}}}{\lambda_{2}} \nabla \cdot \boldsymbol{\tau}_{2}=\mathbf{0} \\
\frac{\boldsymbol{\tau}_{2}-\mathbf{I}}{\lambda_{2}}+\frac{\partial \boldsymbol{\tau}_{2}}{\partial t}+\left(\mathbf{U}_{2} \cdot \nabla\right) \boldsymbol{\tau}_{2}-\left(\nabla \mathbf{U}_{2}\right) \boldsymbol{\tau}_{2}-\boldsymbol{\tau}_{2}\left(\nabla \mathbf{U}_{2}\right)^{t}=\mathbf{0}
\end{array}\right.
$$

where $\mathbf{D}_{i}$ is the rate of deformation tensor of fluid $i, \mathbf{U}_{i}$ is the velocity of fluid $i, p_{i}$ is the pressure of fluid $i, \eta_{1}$ is the dynamic viscosity of fluid $1, \eta_{2}$ is the dynamic viscosity of solvent of fluid $2, \tau_{2}$ is the conformation tensor of fluid 2 , $\lambda_{2}$ is the relaxation time of fluid 2 and $\eta_{p_{2}}$ is the polymer contribution to the solution viscosity.

To complete the model of two-phase flow, boundary conditions at the interface must be added. We have respectively the continuity of velocity and the balance between the jump of normal stress and the surface tension:

$$
\begin{aligned}
\mathbf{U}_{1} & =\mathbf{U}_{2}, \\
(\boldsymbol{\sigma}-\boldsymbol{\delta}) \cdot \mathbf{n} & =\gamma \kappa \mathbf{n}
\end{aligned}
$$

with $\mathbf{n}$ the unit normal vector to the interface, $\kappa$ the curvature of the interface, $\boldsymbol{\sigma}$ and $\boldsymbol{\delta}$ the total stress tensors of fluid 1 and fluid 2 respectively, defined as follows:

$$
\begin{gathered}
\boldsymbol{\sigma}=-p_{1} \mathbf{I}+2 \eta_{1} \mathbf{D}_{1}, \\
\boldsymbol{\delta}=-p_{2} \mathbf{I}+2 \eta_{2} \mathbf{D}_{2}+\frac{\eta_{p 2}}{\lambda_{2}}\left(\boldsymbol{\tau}_{2}-\mathbf{I}\right) .
\end{gathered}
$$

\subsection{The level-set method}

Many numerical techniques have been developed to precisely locate the interface between two fluids [51, 37, 39]. A review of the theory, numerical approximations and applications of the level-set method used in this work can be found in [43, 32]. In the level-set method, the interface $\Sigma$ is represented by the zero isocontour of a signed distance function denoted by $\phi$ called the level-set function (see Figure 1). Hence, at time $t$

$$
\Sigma(t)=\{\mathbf{x}=(x, y) \mid \phi(\mathbf{x}, t)=0\},
$$

where

$$
\phi(\mathbf{x}, t)=\left\{\begin{array}{llc}
-d(\mathbf{x}) & \text { if } & \mathbf{x} \in \Omega_{1} \\
+d(\mathbf{x}) & \text { if } & \mathbf{x} \in \Omega_{2}
\end{array}\right.
$$


and $d(\mathbf{x})$ is the Euclidean distance from the point $\mathbf{x}=(x, y)$ to the interface $\Sigma$, such that for all $\mathbf{x}_{\Sigma} \in \Sigma$, we have

$$
d(\mathbf{x})=\left\{\begin{array}{lll}
-\min \left|\mathbf{x}-\mathbf{x}_{\Sigma}\right| & \text { if } & \mathbf{x} \in \Omega_{1}, \\
+\min \left|\mathbf{x}-\mathbf{x}_{\Sigma}\right| & \text { if } & \mathbf{x} \in \Omega_{2} .
\end{array}\right.
$$

The level-set function $\phi$ is a variety of iso-contours where the interface $\Sigma$ is

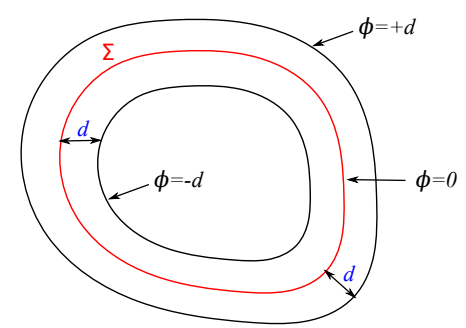

Figure 1: Level-Set function

defined implicitly. Each point of the domain knows its distance to the interface and the phase to which it belongs thanks to the sign of $\phi$. In other words, we have

$$
\left\{\begin{array}{lll}
\phi(\mathbf{x}, t)<0 & \text { if } & \mathbf{x} \in \Omega_{1} \\
\phi(\mathbf{x}, t)>0 & \text { if } & \mathbf{x} \in \Omega_{2} \\
\phi(\mathbf{x}, t)=0 & \text { if } & \mathbf{x} \in \Sigma .
\end{array}\right.
$$

The discontinuous functions $\eta(\phi), \eta_{p}(\phi)$ and $\lambda(\phi)$ are defined by

$$
\begin{aligned}
\eta(\phi) & =\eta_{1}+\left(\eta_{2}-\eta_{1}\right) H(\phi), \\
\eta_{p}(\phi) & =\eta_{p 2} H(\phi), \\
\lambda(\phi) & =\lambda_{2} H(\phi),
\end{aligned}
$$

where $H(\phi)$ is the Heaviside function.

The evolution of the level-set function $\phi$ is governed by the equation:

$$
\frac{\partial \phi}{\partial t}+\mathbf{U} \cdot \nabla \phi=0
$$

where $\mathbf{U}$ is the flow velocity. The unit normal to the interface $\mathbf{n}$, drawn from $\Omega_{1}$ to $\Omega_{2}$, and the curvature $\kappa$ can be expressed in terms of $\phi$ :

$$
\mathbf{n}=\frac{\nabla \phi}{|\nabla \phi|}, \quad \kappa=\nabla \cdot \mathbf{n} .
$$

Abrupt changes of $\eta, \eta_{p}$ and $\lambda$ across the interface could cause numerical difficulties. A regularization of these quantities is performed by introducing a smoothed Heaviside function $H_{\epsilon}$ defined as follows,

$$
H_{\epsilon}(\phi)= \begin{cases}0 & \text { if } \phi<-\epsilon, \\ \frac{1}{2}\left[1+\frac{\phi}{\epsilon}+\frac{1}{\pi} \sin \left(\frac{\pi \phi}{\epsilon}\right)\right] & \text { if }|\phi| \leq \epsilon, \\ 1 & \text { if } \phi>\epsilon,\end{cases}
$$


where $\epsilon$ is a parameter that determines the thickness of the interface. Typically if $\Delta x$ is the mesh size, $\epsilon \in[\Delta x, 2 \Delta x]$. By replacing $H(\phi)$ by $H_{\epsilon}(\phi)$ in $(23),(24)$ and (25), we obtain the smoothed versions of $\eta, \eta_{p}$ and $\lambda$ respectively:

$$
\begin{aligned}
\eta(\phi) & =\eta_{1}+\left(\eta_{2}-\eta_{1}\right) H_{\epsilon}(\phi) \\
\eta_{p}(\phi) & =\eta_{p 2} H_{\epsilon}(\phi) \\
\lambda(\phi) & =\lambda_{2} H_{\epsilon}(\phi) .
\end{aligned}
$$

Using the level-set function, the system of governing equations for the two fluids (13) and (14) with the boundary conditions at the interface (15) and (16) can be written as a system of equations valid in the whole domain $\Omega$ as follows:

$$
\left\{\begin{aligned}
\nabla \cdot \mathbf{U}=0 & \text { in } \quad(0, T) \times \Omega \\
-\nabla p+\nabla \cdot(2 \eta(\phi) \mathbf{D})+\nabla \cdot\left(\frac{\eta_{p}(\phi)}{\lambda(\phi)} \boldsymbol{\tau}\right)-\gamma \kappa(\phi) \delta(\phi) \frac{\nabla \phi}{|\nabla \phi|}=\mathbf{0} & \text { in } \quad(0, T) \times \Omega \\
\frac{\boldsymbol{\tau}-\mathbf{I}}{\lambda(\phi)}+\frac{\partial \boldsymbol{\tau}}{\partial t}+(\mathbf{U} \cdot \nabla) \boldsymbol{\tau}-(\nabla \mathbf{U}) \boldsymbol{\tau}-\boldsymbol{\tau}(\nabla \mathbf{U})^{t}=\mathbf{0} & \text { in } \quad(0, T) \times \Omega \\
\frac{\partial \phi}{\partial t}+\mathbf{U} \cdot \nabla \phi=0 & \text { in } \quad(0, T) \times \Omega
\end{aligned}\right.
$$

where $\delta(\phi)$ is the Dirac function. In the momentum equation, the surface tension force is interpreted as a body force localized on the interface. The derivation of this formulation can be found in [8].

\subsection{Cox model interpretation}

We denote by $\theta_{d}$ the angle between the fluid/fluid interface and the solid wall, by $\theta_{a}$ the advancing critical angle and by $\theta_{r}$ the receding critical angle. The angles $\theta_{a}$ and $\theta_{r}$ are known parameters in our simulations, they depend on several factors such as the nature of fluids and the solid surface. During the simulation, the interface is deformed by the flow and the angle $\theta_{d}$ changes. The dynamic of the triple line is modeled as follows:

- when the angle $\theta_{d}$ exceeds the angle $\theta_{a}$, the triple line moves with a velocity $V_{\text {cox }}$ towards the fluid 1 ,

- on the other hand, if $\theta_{d}$ becomes smaller than $\theta_{r}$, the triple line moves with a velocity $V_{c o x}$ towards the fluid 2,

- otherwise, the triple line does not move.

Figure 2 shows a triple line in the three cases described above. According to this model, the triple line remains steady when $\theta_{r}<\theta_{d}<\theta_{a}$ and it moves when $\theta_{d}<\theta_{r}$ or $\theta_{d}>\theta_{a}$. If $\theta_{r}<\theta_{d}<\theta_{a}$, the boundary condition at the solid wall is a no-slip condition. If the triple line is allowed to move, the no-slip boundary condition is replaced by a slip condition in the vicinity of the triple line. The velocity of the triple line is denoted $V_{c o x}$ and is calculated from the equation (9). 


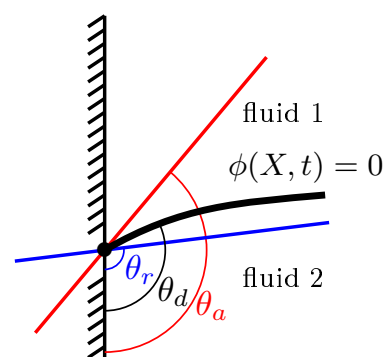

(a) $\theta_{r}<\theta_{d}<\theta_{a}$ The triple line remains steady.

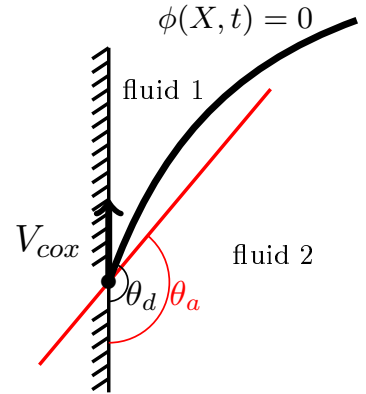

(b) $\theta_{d}>\theta_{a}$.

The triple line moves towards the fluid 1.

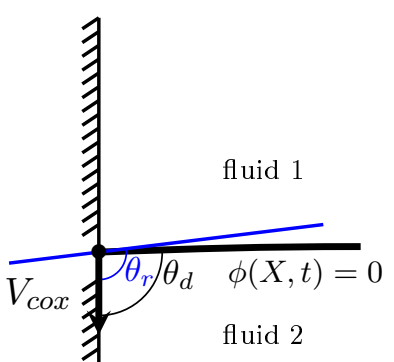

(c) $\theta_{d}<\theta_{r}$

The triple line moves towards the fluid 2.

Figure 2: Cox model interpretation.

\subsection{The penalization method and the dimensionless governing equations}

Figure 3 shows a computational domain with a micro-channels network. This network is formed of micro-channels with different diameters separated by nonuniform distances. In order to overcome the difficulty of creating an adaptive mesh to the geometry, the volume penalization method [2] is used. Therefore, the boundary conditions on the immersed boundaries in the domain are taken into account by adding penalization terms in the equations (32). Indeed, let $\Omega$

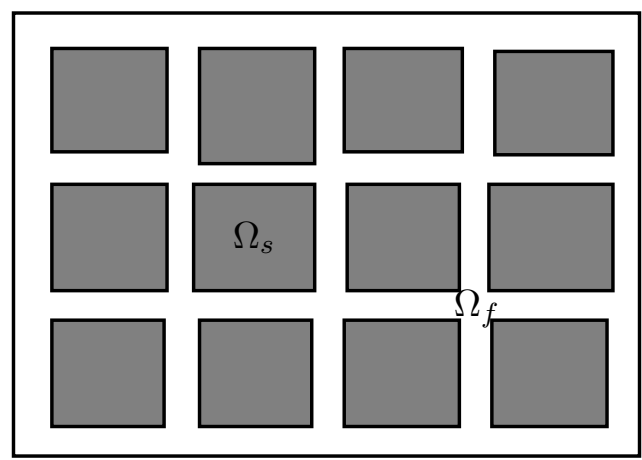

Figure 3: Domain $\Omega=\Omega_{f} \cup \Omega_{s}$

be the domain shown in the Figure 3 , we denote by $\Omega_{f}$ the fluid part and $\Omega_{s}$ the reunion of solid obstacles. Instead of solving the system of equations (32) in $\Omega_{f}$, an equivalent problem is solved in the whole domain $\Omega$ by penalizing the velocity in the solid part. This method consists in adding the terms $\frac{\mathbf{U}}{K}$ and $\frac{\boldsymbol{\tau}-\boldsymbol{\tau}_{s}}{K}$ in the second and third equations of system (32) respectively, where $K$ can be considered as a dimensionless permeability coefficient and $\boldsymbol{\tau}_{s}$ is the identity tensor. The value of $K$ is set very large in the fluid zone (e.g. $10^{16}$ or more) and very small in the solid part (e.g. $\left.10^{-8}\right)$. In consequence, the initial Stokes equations are recovered in the fluid part as the extra term $\frac{\mathrm{U}}{K}$ vanishes and the velocity is of the order $10^{-8}$ in the solid part (see [2] for more details). So the 
velocity is negligeable and the solid part is recovered. In the tensor equation, it is not necessary to add the term $\frac{\boldsymbol{\tau}-\boldsymbol{\tau}_{s}}{K}$ to penalize the tensor in the solid parts. However, results are more stable as one of the main difficulties of the numerical simulations is to capture the strong gradients in the vicinity of the solid walls [55].

To put the system of equations into dimensionless form, we choose the following scaling:

$$
\begin{gathered}
x=L_{c} x^{\prime}, y=L_{c} y^{\prime}, t=\frac{L_{c}}{U_{c}} t^{\prime}, \\
p=\frac{\eta_{c} U_{c}}{L_{c}} p^{\prime}, \mathbf{U}=U_{c} \mathbf{U}^{\prime}, \eta=\eta_{c} \eta^{\prime}, \eta_{p}=\eta_{c} \eta_{p}^{\prime}, \lambda=\frac{L_{c}}{U_{c}} \lambda^{\prime},
\end{gathered}
$$

where the prime variables are the dimensionless quantities, $L_{c}$ is the characteristic length, $U_{c}$ the characteristic velocity and $\eta_{c}$ the characteristic viscosity. In practice, the characteristic velocity is the injection velocity and the characteristic length is the diameter of the channel where the viscoelastic fluid is injected. The characteristic viscosity will be always $\eta_{1}$, the dynamic viscosity of Newtonian fluid 1. By introducing these dimensionless variables without the prime for the sake of simplicity, the system of equations becomes on the whole domain $\Omega$ :

$$
\left\{\begin{aligned}
\nabla \cdot \mathbf{U} & =0 \\
-\nabla p+\nabla \cdot(2 \eta(\phi) \mathbf{D})+\nabla \cdot\left(\frac{\eta_{p}(\phi)}{\lambda(\phi)} \boldsymbol{\tau}\right)-\frac{1}{C a} \kappa(\phi) \delta(\phi) \frac{\nabla \phi}{|\nabla \phi|}+\frac{\mathbf{U}}{K} & =\mathbf{0} \\
\frac{\boldsymbol{\tau}-\mathbf{I}}{\lambda(\phi)}+\frac{\partial \boldsymbol{\tau}}{\partial t}+(\mathbf{U} \cdot \nabla) \boldsymbol{\tau}-(\nabla \mathbf{U}) \boldsymbol{\tau}-\boldsymbol{\tau}(\nabla \mathbf{U})^{t}+\frac{\boldsymbol{\tau}-\boldsymbol{\tau}_{s}}{K} & =\mathbf{0} \\
\frac{\partial \phi}{\partial t}+\mathbf{U} \cdot \nabla \phi & =0
\end{aligned}\right.
$$

where $C a$ is the capillary number defined by $C a=\frac{\eta_{c} U_{c}}{\gamma}$. The dimensionless viscosities and the dimensionless relaxation time are:

$$
\begin{aligned}
\eta^{\prime}(\phi) & =1+\left(\frac{\eta_{2}}{\eta_{c}}-1\right) H_{\epsilon}(\phi), \\
\eta_{p}^{\prime}(\phi) & =\frac{\eta_{p 2}}{\eta_{c}} H_{\epsilon}(\phi), \\
\lambda^{\prime}(\phi) & =\lambda_{2}^{\prime} H_{\epsilon}(\phi) .
\end{aligned}
$$

\subsection{Initial and boundary conditions}

Let $\Omega$ be the bounded domain in $\mathbb{R}^{2}$ with boundary $\partial \Omega$, we denote $\partial \Omega_{i}$ the inlet boundary where a constant velocity is imposed, $\partial \Omega_{p}$ the part of boundary where periodic conditions are specified and $\partial \Omega_{o}$ the outlet boundary where artificial non-reflecting boundary conditions are imposed [6]. 
The initial conditions for each variable in the system (33) are:

$$
\left\{\begin{aligned}
u(\mathbf{x}, 0) & =0 & & \forall \mathbf{x} \in \Omega \\
v(\mathbf{x}, 0) & =v_{i n j} & & \forall \mathbf{x} \in \partial \Omega_{i} \\
\tau_{x x}(\mathbf{x}, 0) & =1 & & \forall \mathbf{x} \in \Omega_{f} \\
\tau_{x x}(\mathbf{x}, 0) & =0 & & \forall \mathbf{x} \in \Omega_{s} \\
\tau_{x y}(\mathbf{x}, 0) & =0 & & \forall \mathbf{x} \in \Omega \\
\tau_{y y}(\mathbf{x}, 0) & =1 & & \forall \mathbf{x} \in \Omega_{f} \\
\tau_{y y}(\mathbf{x}, 0) & =0 & & \forall \mathbf{x} \in \Omega_{s} \\
\phi(\mathbf{x}, 0) & =\phi_{0} & & \forall \mathbf{x} \in \Omega
\end{aligned}\right.
$$

where $v_{i n j}$ is the constant injection velocity.

At inlet $\partial \Omega_{i}$, the injection velocity is prescribed for all times:

$$
\left\{\begin{array}{l}
\left.u\right|_{\partial \Omega_{i}}=0 \\
\left.v\right|_{\partial \Omega_{i}}=v_{i n j}
\end{array}\right.
$$

Substituting (38) in the conformation tensor equation of system (33), yields:

$$
\left\{\begin{array}{l}
\left.\tau_{x x}\right|_{\partial \Omega_{i}}=1 \\
\left.\tau_{x y}\right|_{\partial \Omega_{i}}=0 \\
\left.\tau_{y y}\right|_{\partial \Omega_{i}}=1
\end{array}\right.
$$

If ( $\left.\mathbf{U}^{r e f}, p^{r e f}\right)$ is a referential solution in $\Omega$, the artificial non-reflecting boundary condition on $\partial \Omega_{o}$ for the Newtonian fluid 1 reads:

$$
\boldsymbol{\sigma}(\mathbf{U}, p) \mathbf{n}=\boldsymbol{\sigma}\left(\mathbf{U}^{r e f}, p^{r e f}\right) \mathbf{n},
$$

where $\boldsymbol{\sigma}$ is the total stress tensor for the Newtonian fluid defined in (17) and $\mathbf{n}$ is the outward unit normal vector to the domain. For the viscoelastic fluid 2, the boundary condition on $\partial \Omega_{o}$ is

$$
\left\{\begin{array}{l}
\boldsymbol{\delta}(\mathbf{U}, p, \boldsymbol{\tau}) \mathbf{n}=\boldsymbol{\delta}\left(\mathbf{U}^{r e f}, p^{r e f}, \boldsymbol{\tau}^{r e f}\right) \mathbf{n}, \\
\boldsymbol{\tau}-\boldsymbol{\tau}^{r e f}=0
\end{array}\right.
$$

where $\boldsymbol{\delta}$ is the total stress tensor for fluid 2 defined in (18). In these conditions $\left(\mathbf{U}^{r e f}, p^{r e f}\right)$ can be for instance a Poiseuille flow in a channel and $\boldsymbol{\tau}^{r e f}$ is the resulting tensor computed with this choice.

\section{Numerical resolution}

\subsection{Time discretization}

Let $\left(\mathbf{U}^{n}, p^{n}, \boldsymbol{\tau}^{n}, \phi^{n}\right)$ be the approximation of $(\mathbf{U}, p, \boldsymbol{\tau}, \phi)$ at time $t^{n}=n \Delta t$ where $n \in \mathbb{N}$ and $\Delta t$ is the time step, $\left(\mathbf{U}^{n+1}, p^{n+1}\right)$ are computed first, then $\boldsymbol{\tau}^{n+1}$ and 
finally $\phi^{n+1}$. In Stokes equations, the terms $\frac{1}{C a} \kappa(\phi) \delta(\phi) \nabla \phi$ and $\nabla \cdot \boldsymbol{\tau}$ are treated explicitly. Therefore the semi discretized equations read:

$$
\begin{aligned}
\nabla \cdot \mathbf{U}^{n+1} & =0, \\
-\nabla p^{n+1}+\nabla \cdot\left(2 \eta\left(\phi^{n}\right) \mathbf{D}\left(\mathbf{U}^{n+1}\right)\right)+\frac{\mathbf{U}^{n+1}}{K}= & \frac{1}{C a} \kappa\left(\phi^{n}\right) \delta\left(\phi^{n}\right) \nabla \phi^{n} \\
& -\nabla \cdot\left(\frac{\eta_{p}\left(\phi^{n}\right)}{\lambda\left(\phi^{n}\right)} \boldsymbol{\tau}^{n}\right) .
\end{aligned}
$$

The conformation tensor equation is solved in two half-steps. First, the advection term is taken into account,

$$
\frac{\boldsymbol{\tau}^{n+1 / 2}-\boldsymbol{\tau}^{n}}{\Delta t}+\left(\mathbf{U}^{n+1} \cdot \nabla\right) \boldsymbol{\tau}^{n}=\mathbf{0}
$$

then the others terms are considered:

$$
\frac{\left(\boldsymbol{\tau}^{n+1}-\mathbf{I}\right)}{\lambda\left(\phi^{n}\right)}+\frac{\boldsymbol{\tau}^{n+1}-\boldsymbol{\tau}^{n+1 / 2}}{\Delta t}-\left(\nabla \mathbf{U}^{n+1}\right) \boldsymbol{\tau}^{n+1}-\boldsymbol{\tau}^{n+1}\left(\nabla \mathbf{U}^{n+1}\right)^{T}+\frac{\boldsymbol{\tau}^{n+1}}{K}=\mathbf{0} .
$$

The level-set transport equation is discretized in time by the explicit Euler scheme:

$$
\frac{\phi^{n+1}-\phi^{n}}{\Delta t}+\left(\mathbf{U}^{n+1} \cdot \nabla\right) \phi^{n}=\mathbf{0} .
$$

Solving Stokes equations with an explicit treatment of the term associated to the surface tension requires a stability criterion. In this work, the stability condition proposed in [19] is used:

$$
\Delta t \leq c \frac{\min \left(\eta_{1}, \eta_{2}\right)}{\gamma} \Delta x
$$

The stability constraint (46) can be very restrictive when the velocity of the flow is very low compared to $\frac{\gamma}{\eta}$. Thus, if the numerical method leads to smoothed curvature, the constant $c$ can be of an order greater than one [19]. After some numerical tests, we found that $c=6$ is the critical value for which the computation is always stable.

\subsection{Space discretization}

\subsubsection{Computation of $\left(\mathbf{U}^{n+1}, p^{n+1}\right)$}

Let $\Delta x$ and $\Delta y$ be the space steps on a rectangular Cartesian grid, the points $\left(x_{i}, y_{j}\right)$ are the cell centers defined by

$$
\left(x_{i}, y_{j}\right)=\left(\left(i-\frac{1}{2}\right) \Delta x,\left(j-\frac{1}{2}\right) \Delta y\right) \text { for } i=1, \ldots, N x \text { and } j=1, \ldots, N y .
$$

The pressure unknowns $p$ are located at these centers while the velocity components $u$ and $v$ are located at the middle of the edges of the cells as shown in Figure 4.

The system (42) is transformed into a constrained minimization problem solved 


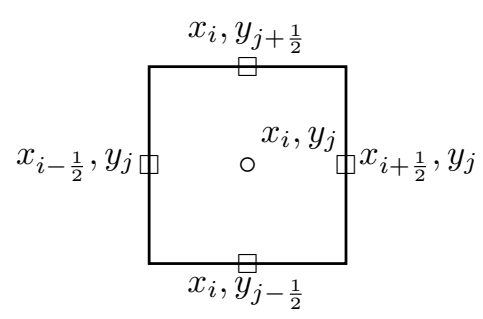

(a) Points

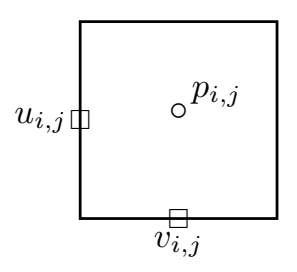

(b) Unknowns

Figure 4: Notations of points and unknowns on a staggered cell.

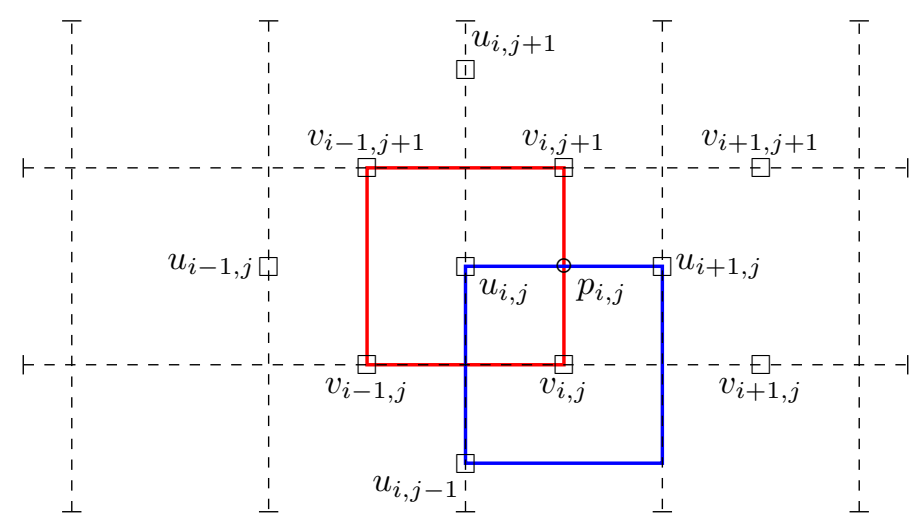

Figure 5: Control volumes $V_{u}$ (red), $V_{v}$ (blue), $V_{p}$ (dotted lines)

by the augmented Lagrangian method [18]. Thus $\left(\mathbf{U}^{n+1}, p^{n+1}\right)$ is obtained using Uzawa algorithm with $1 \leq k \leq L$ :

$$
\begin{aligned}
& -\nabla p^{n+\frac{k-1}{L}}+\nabla \cdot\left(2 \eta^{n} \mathbf{D}\left(\mathbf{U}^{n+\frac{k}{L}}\right)\right)+r_{1} \nabla\left(\nabla \cdot \mathbf{U}^{n+\frac{k}{L}}\right)+\frac{\mathbf{U}^{n+\frac{k}{L}}}{K}= \\
& \frac{1}{C a} \kappa\left(\phi^{n}\right) \delta\left(\phi^{n}\right) \nabla \phi^{n}-\frac{\eta_{p}\left(\phi^{n}\right)}{\lambda\left(\phi^{n}\right)} \nabla \cdot \boldsymbol{\tau}^{n}, \\
& p^{n+\frac{k}{L}}=p^{n+\frac{k-1}{L}}-r_{2}\left(\nabla \cdot \mathbf{U}^{n+\frac{k}{L}}\right),
\end{aligned}
$$

where $r_{1}$ and $r_{2}$ are the coefficients of the augmented Lagrangian method.

Let $V_{u}$ the control volume centered in $u, V_{v}$ be the control volume centered in $v$ and $V_{p}$ be the control volume centered in $p$ (see Figure 5), the edges of $V_{u}$ and $V_{v}$ are denoted $\partial V_{u}$ and $\partial V_{v}$ respectively with the notations:

$$
\begin{aligned}
& \partial V_{u}=\partial V_{u}^{x} \cup \partial V_{u}^{y}=\partial V_{u}^{x+} \cup \partial V_{u}^{x-} \cup \partial V_{u}^{y+} \cup \partial V_{u}^{y-} \\
& \partial V_{v}=\partial V_{v}^{x} \cup \partial V_{v}^{y}=\partial V_{v}^{x+} \cup \partial V_{v}^{x-} \cup \partial V_{v}^{y+} \cup \partial V_{v}^{y-}
\end{aligned}
$$

where $V_{u}^{x+}$ is the edge oriented in the positive direction of $x$.

The equation for $u$ in (47) is integrated over the control volume $V_{u}$ using the divergence theorem (the upper index are omitted) and writting $\delta$ as the Heaviside derivative [52]. 


$$
\begin{aligned}
& -\int_{V_{u}} \frac{\partial p}{\partial x} d x d y+\int_{V_{u}^{x^{+}}} 2 \eta \frac{\partial u}{\partial x} d y-\int_{V_{v^{x^{-}}}} 2 \eta \frac{\partial u}{\partial x} d y+\int_{V_{u}^{y^{+}}} \eta\left(\frac{\partial u}{\partial y}+\frac{\partial v}{\partial x}\right) d x \\
& -\int_{V_{u}^{y^{-}}} \eta\left(\frac{\partial u}{\partial y}+\frac{\partial v}{\partial x}\right) d x+r_{1} \int_{V_{u}^{x^{+}}}\left(\frac{\partial u}{\partial x}+\frac{\partial v}{\partial y}\right) d y-r_{1} \int_{V_{u}^{x^{-}}}\left(\frac{\partial u}{\partial x}+\frac{\partial v}{\partial y}\right) d y \\
& +\int_{V_{u}} \frac{u}{K} d x d y=\int_{V_{u}} \frac{1}{C a} \kappa(\phi) \frac{\partial H(\phi)}{\partial x} d x d y-\int_{V_{u}} \frac{\eta_{p}}{\lambda}\left(\frac{\partial \tau_{x x}}{\partial x}+\frac{\partial \tau_{x y}}{\partial y}\right) d x d y
\end{aligned}
$$

where

$$
\begin{aligned}
\int_{V_{u}} \frac{1}{C a} \kappa(\phi) \frac{\partial H}{\partial x}(\phi) d x d y & =\frac{1}{C a} \int_{V_{u}} \nabla \cdot \mathbf{n} \frac{\partial H}{\partial x}(\phi) d x d y \\
& \approx \frac{1}{C a} \frac{\int_{V_{u}} \nabla \cdot \mathbf{n} d x d y}{\Delta x \Delta y} \int_{V_{u}} \frac{\partial H}{\partial x}(\phi) d x d y
\end{aligned}
$$

with

$$
\int_{V_{u}} \frac{\partial H}{\partial x}(\phi) d x d y \approx \Delta y\left(H_{\epsilon}\left(\phi_{i, j}\right)-H_{\epsilon}\left(\phi_{i-1, j}\right)\right)
$$

and

$$
\int_{V_{u}} \nabla \cdot \mathbf{n} d x d y=\int_{\partial V_{u}} \mathbf{n} \cdot \mathbf{n}_{u} d s
$$

$\mathbf{n}_{u}$ being the unit outward normal to $V_{u}$.

Finally, using the second order approximation $\int_{V_{c}} q d x d y \approx \bar{q} \Delta x \Delta y$, the finite volume scheme for $u$ integrated over the control volume $V_{u}$ is:

$$
\begin{aligned}
& -\left(p_{i, j}-p_{i-1, j}\right) \Delta y+\left(2 \eta_{i, j} \frac{u_{i+1, j}-u_{i, j}}{\Delta x}-2 \eta_{i-1, j} \frac{u_{i, j}-u_{i-1, j}}{\Delta x}\right) \Delta y \\
& +\eta_{i, j+1}^{u, y}\left(\frac{u_{i, j+1}-u_{i, j}}{\Delta y}+\frac{v_{i, j+1}-v_{i-1, j+1}}{\Delta x}\right) \Delta x-\eta_{i, j}^{u, y}\left(\frac{u_{i, j}-u_{i, j-1}}{\Delta y}+\frac{v_{i, j}-v_{i-1, j}}{\Delta x}\right) \Delta x \\
& +r_{1}\left(\frac{u_{i+1, j}-u_{i, j}}{\Delta x}+\frac{v_{i, j+1}-v_{i, j}}{\Delta y}\right) \Delta y-r_{1}\left(\frac{u_{i, j}-u_{i-1, j}}{\Delta x}+\frac{v_{i-1, j+1}-v_{i, j}}{\Delta y}\right) \Delta y+\frac{u_{i, j}}{K} \\
& =\frac{1}{C a} \frac{1}{\Delta x \Delta y}\left(\left(n_{i, j}^{x}-n_{i-1, j}^{x}\right) \Delta y+\left(\tilde{n}_{i, j+1}^{y}-\tilde{n}_{i, j}^{y}\right) \Delta x\right) \Delta y\left(H\left(\phi_{i, j}\right)-H\left(\phi_{i-1, j}\right)\right) \\
& -\frac{\eta_{p_{i, j}}^{u}}{\lambda_{i, j}^{u}}\left(\tau_{x x_{i, j}}-\tau_{x x_{i-1, j}}\right) \Delta y-\frac{\eta_{p_{i, j}}^{u}}{\lambda_{i, j}^{u}}\left(\tau_{x x_{i, j+1}}-\tau_{x y_{i, j}}\right) \Delta x,
\end{aligned}
$$

where the diagonal components of the tensor $\tau_{x x}$ and $\tau_{y y}$ are located at the centers of the cell like $p$ and the extra diagonal components $\tau_{x y}$ are located at the vertices. The equation for $v$ is derived in the same way.

\subsubsection{Computation of $\tau^{n+1}$ and $\phi^{n+1}$}

Once the velocity and pressure are updated, the conformation tensor is computed in two steps as said above in section 4.1. The first step consists in solving the advection equation (43) discretized using WENO-5 scheme (Weithed Essentially Non Oscillatory scheme of fifth order) as detailed in [30, 25]. This is done for the three components of the tensor $\tau_{x x}^{n+1 / 2}, \tau_{y y}^{n+1 / 2}$ and $\tau_{x y}^{n+1 / 2}$. With staggered grids, it is necessary to determine the other unknowns at the proper locations, 
which is done by linear interpolations.

Then, the second step solving equation (44) is performed using the second order centered scheme and linear interpolations when needed. Hence the unknowns $\tau_{x x}^{n+1}, \tau_{y y}^{n+1}$ and $\tau_{x y}^{n+1}$ at time $(n+1) \Delta t$ are updated.

Finally, the equation (45) is discretized once again using WENO-5 scheme on a twice finer grid in each direction to get $\phi^{n+1}$ as accurately as possible. The unknown $\phi$ is located at the vertices of this finer grid and denoted $\tilde{\phi}$. Besides the values of the velocity are interpolated linearly on the finer grid.

Practical experience suggests that level-set methods are sensitive to spatial accuracy, whereas temporal truncation errors seem to produce significantly less deterioration of the numerical solution. In consequence, one can often use the fifth-order accurate WENO method for spatial discretization and first-order Euler method for discretization in time [32]. Although it is shown in [54] that this time integration leads to instabilities, we do not observe any oscillations as the interface moves very slowly. Indeed, for our microfluidics applications, the velocity is very low and the time step is also very low due to the surface tension term.

\subsection{Redistanciation of the Level-Set}

The transport equation of the Level-Set function (26) was built such that the function $\phi$ is advected by the flow velocity $\mathbf{U}$. Unless the velocity $\mathbf{U}$ is the same for all contour lines, this procedure implies that during the time iterations $|\nabla \phi| \neq 1$. In consequence, the Level-Set function loses its signed distance property leading to erroneous calculations of the normal vector to the interface or numerical instabilites in the areas where $\phi$ presents strong gradients. For this reason, it is necessary to apply a redistanciation procedure to the Level-Set function to recover the signed distance property. The need to do so in level-set calculations is exposed in [10]. The main idea is to periodically recalculate the function $\phi$ (here every twenty time step) without altering the position of the interface. In this work, the Fast Marching method, presented in [42], is used for redistanciating the Level-Set function. At the initialisation, the narrow band is defined as the positive points with at least one horizontal or vertical negative neighbor. The known points are the negative points.

\subsection{Triple lines evolution}

One of the main part of this work is the detection of triple lines and their evolution using Cox law. Here we have to take into account the penalization method used to represent the solid parts inside the computational domain $\Omega$. To illustrate the problem let us have a look to the flow inside a short symmetrical network shown in Figure 6. One can see clearly the fluid 2 pushing the fluid 1 and the interface between the two fluids which is the zero value of the level-set function. The interface at the initialization is an horizontal straight line at the entrance section that is pushed by fluid 2 and follows the solid parts. In this example, there are six triple lines, two in each channel. They have first to be detected and then they must remain steady or move according to the contact angle as 
explained in section 3.3.

A triple point is detected when the signs of the level-set function of two neigh-

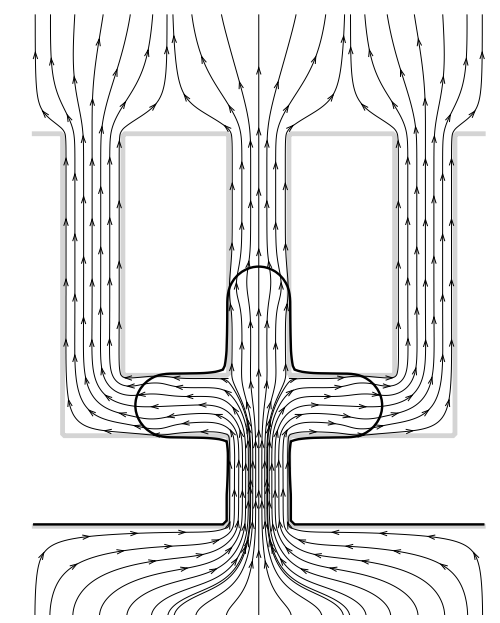

Figure 6: Example of flow interface in a short symmetrical network. The fluid 2 enters at the bottom and pushes the fluid 1 already present inside the network.

bouring solid points are opposite. The triple point is thus in between. This detection must be done in accordance to the direction of the boundary of the solid parts and to the direction of the flow (see Figure 7). Once a triple point is detected it is necessary to compute the contact angle at this point. The first step

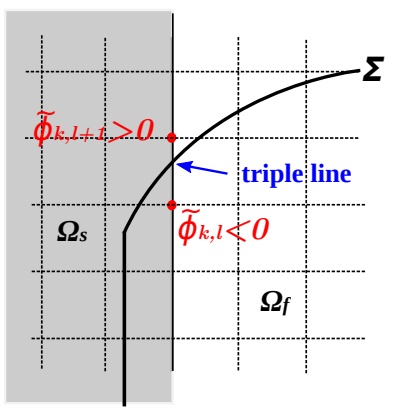

(a) Vertical left boundary

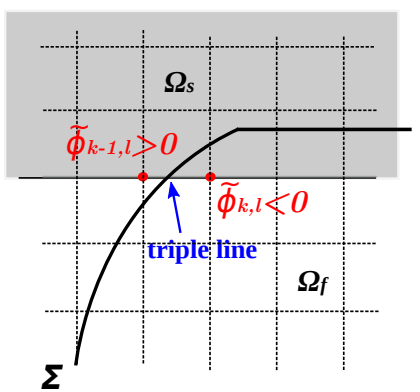

(b) Horizontal right boundary

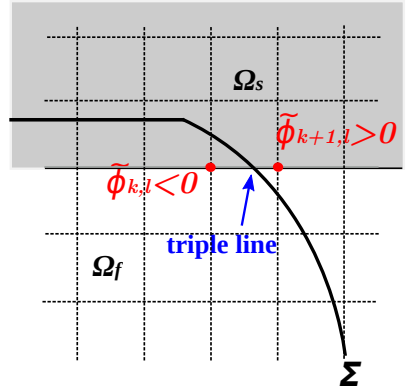

(c) Horizontal left boundary

Figure 7: Triple points detection with respect to the flow direction. The solid parts are colored in grey.

is to compute the normal vector at the triple point. For instance, if this point is located between points $\left(x_{k}, y_{l}\right)$ and $\left(x_{k}, y_{l+1}\right)$ as in Figure 7 (a), the components of this normal vector $\tilde{\mathbf{n}}^{*}=\left(\tilde{n}^{x^{*}}, \tilde{n}^{y^{*}}\right)$ on the finer grid are determined by linear interpolation:

$$
\tilde{n}^{x^{*}}=\tilde{n}_{k, l}^{x}-\tilde{\phi}_{k, l} \frac{\tilde{n}_{k, l+1}^{x}-\tilde{n}_{k, l}^{x}}{\tilde{\phi}_{k, l+1}-\tilde{\phi}_{k, l}}, \quad \tilde{n}^{y^{*}}=\tilde{n}_{k, l}^{y}-\tilde{\phi}_{k, l} \frac{\tilde{n}_{k, l+1}^{y}-\tilde{n}_{k, l}^{y}}{\tilde{\phi}_{k, l+1}-\tilde{\phi}_{k, l}} .
$$

where $\tilde{\mathbf{n}}=\left(\tilde{n}_{k, l}^{x}, \tilde{n}_{k, l}^{y}\right)$ denotes the normal vector at point $\left(x_{k}, y_{l}\right)$. Due to the finer grid, the computation of the normal vector at points $\left(x_{k}, y_{l}\right)$ and $\left(x_{k}, y_{l+1}\right)$ 
is done with a smooth level-set function as the singularity occurs inside the solid part. So it does not interfere except at the initialization when there is a flat interface transverse to the channel.

Then the contact angle $\theta_{d}$ is computed from the normal $\tilde{\mathbf{n}}^{*}$ as shown in Figure 8 :

$$
\sin (\alpha)=\frac{n^{y^{*}}}{\sqrt{n^{x^{2}}+n^{y^{* 2}}}} \text { and } \theta_{d}=\pi-\alpha
$$

Finally the triple points may move accordingly to Cox law. When the contact

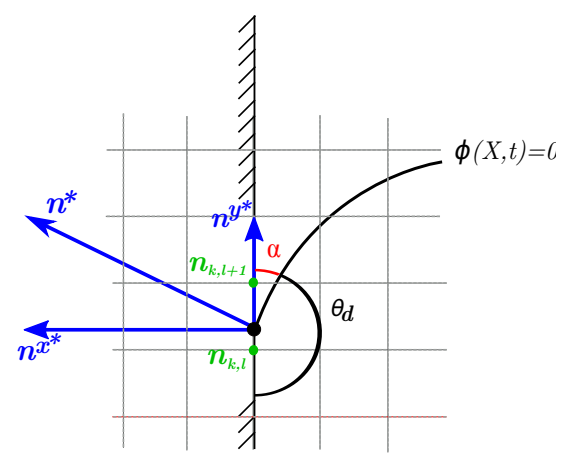

Figure 8: Computation of the contact angle $\theta_{d}$ on a vertical left boundary.

angle $\theta_{d}$ is large enough, the triple point must move with the speed $V_{d}$ and thus Stokes equations are solved with a slip boundary condition in the vicinity $\mathcal{V}$ of the triple point (for instance $\mathcal{V}=\{(k, l-3),(k, l-2),(k, l-1),(k, l),(k, l+1)$, $(k, l+2),(k, l+3)\}$ according to the stencil of WENO-5 scheme. To do that the penalization term is changed to $\frac{\mathrm{U}-V_{d}}{K}$ in the momentum equation with $V_{d}$ given by:

$$
V_{d}= \begin{cases}\sum_{i=1}^{n t r i p l e s}\left(V_{\text {cox }}\right)_{i} \chi_{\mathcal{V}_{i}} & \text { if } \theta_{d}<\theta_{r} \text { or } \theta_{d}>\theta_{a}, \\ 0 & \text { otherwise }\end{cases}
$$

where ntriples is the number of triple points and $\chi_{\mathcal{V}_{i}}$ the characteristic function of the neighbourhood $\mathcal{V}_{i}$ of point $i$ and $V_{c o x}$ is the Cox speed calculated from equation (9).

When a triple point moves along a boundary, the Cox speed is imposed on $\mathcal{V}_{i}$ according to its position on the mesh for Stokes equations as shown on Figure 9 and Figure 10. The speed $V_{c o x}$ is imposed in such a way that the induced flowrate does not change when the point is moving.

\section{Validation test}

In this validation test, we propose to verify the accurate computation of the interface curvature and how it satifies the Laplace's law, which describes that the pressure difference across the interface between two fluids is proportional to the interface curvature with a coefficient equals to the surface tension. By 


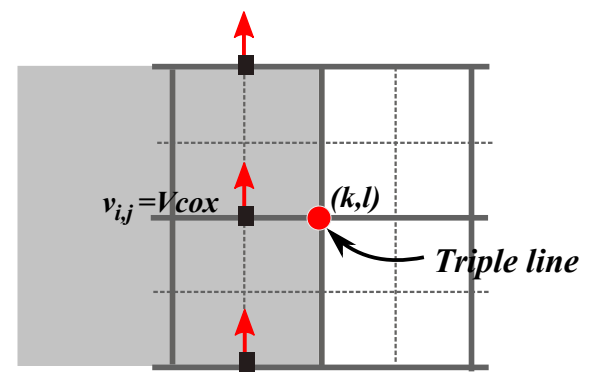

(a)

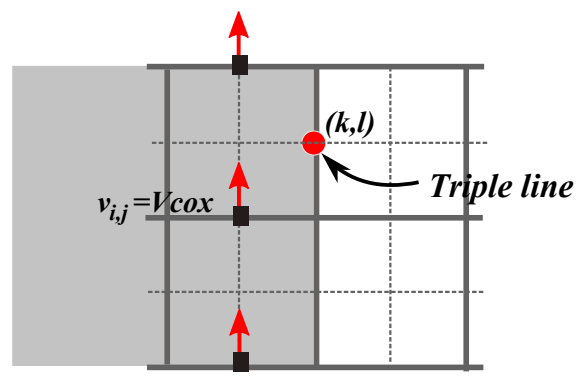

(b)

Figure 9: The triple point moves from a cell corner (a) to a middle of a side (b).

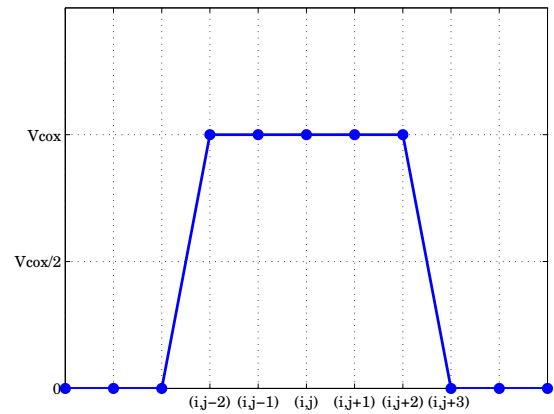

(a)

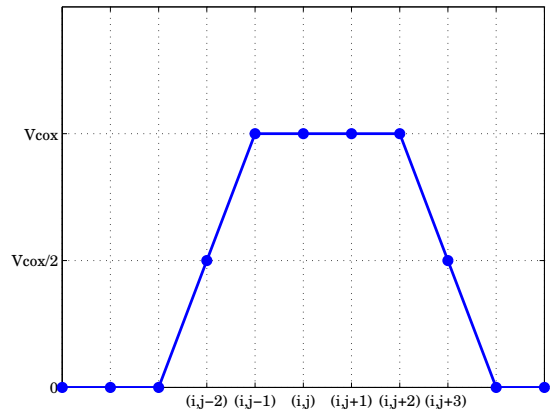

(b)

Figure 10: The speed $V_{\text {cox }}$ when the point moves from the cell corner (a) to the middle of the side (b) as in Figure 9.

considering a circular static drop inside two-dimensional channel, the Laplace's law writes:

$$
\Delta P=\frac{\gamma}{R},
$$

where $\Delta P$ is the pressure difference across the fluid interface, $\gamma$ is the surface tension and $R$ is the radius of curvature of the interface.

The parameters of the simulation are the viscosity inside the drop $\eta_{1}=55 \mathrm{mPa} . \mathrm{s}$, the viscosity of the external fluid $\eta_{2}=305 \mathrm{mPa.s}$, the coefficient of the surface tension $\gamma=32 \mathrm{mN} / \mathrm{m}$ and the radius $R=10 \mu \mathrm{m}$. The injection rate is zero. The theoretical value of the pressure difference given by the equation (51) is $3200 \mathrm{~Pa}$. A mesh convergence study is performed using four different mesh sizes, results are shown in Table 1 . These results prove that the employed method for the curvature computation is very efficient as it is almost second order. 
Table 1: Mesh convergence study of the interface curvature. $\Delta P_{\text {num }}$ is the numerical difference pressure and $E$ is the relative error.

\begin{tabular}{|c|r|c|c|}
\hline Mesh & $\Delta P_{\text {num }}$ & $E$ & Order \\
\hline $64 \times 64$ & 3204.028 & $1.25 \times 10^{-3}$ & \\
$128 \times 128$ & 3201.213 & $3.79 \times 10^{-4}$ & 1.72 \\
$256 \times 256$ & 3200.288 & $9.00 \times 10^{-5}$ & 2.07 \\
$512 \times 512$ & 3200.087 & $2.71 \times 10^{-5}$ & 1.73 \\
\hline
\end{tabular}

\section{Results in a single channel}

\subsection{Newtonian-Newtonian case}

The first numerical test concerns the flow in a straight channel shown in Figure 11. The domain dimensions are $L_{x}$ and $L_{y}$ and the channel diameter is $L_{c}$. The characteristic length is $L_{c}$ while $U_{c}=\frac{Q}{L_{c}}$ is the characteristic velocity. The capillary number is, $C a=\frac{U_{c} \eta_{1}}{\gamma}$ with $\eta_{1}$ the dynamic viscosity of the fluid 1 and $\gamma$ the coefficient of surface tension. The Cox angle $\theta_{a}$ is fixed according to experiments. Indeed, if this angle is set to a too large value, the contact line cannot move, the limit case is $\theta_{a}=180^{\circ}$ that corresponds to the no-slip boundary condition. The first test case is the simulation of a two-phase flow with two

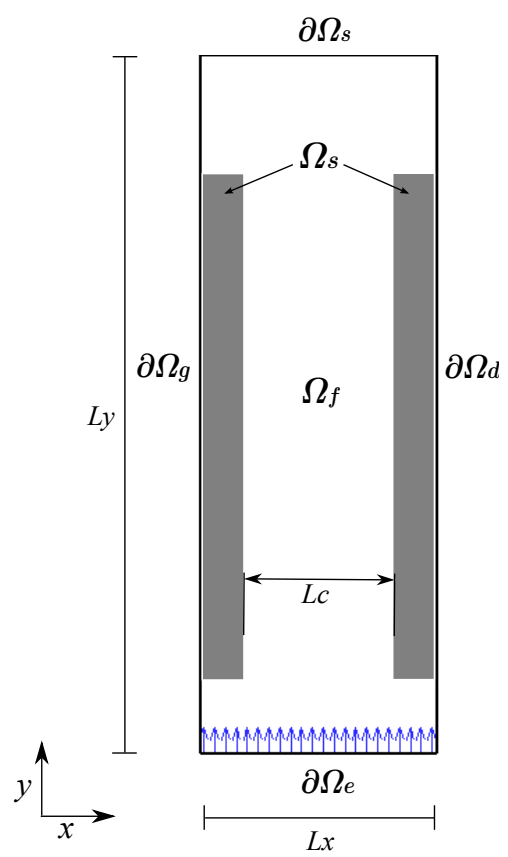

Figure 11: Geometry of the domain for the two-phase flow simulation in a channel. 
Newtonian fluids. The mesh has $96 \times 288$ cells and the physical parameters are:

$$
\left\{\begin{array}{l}
L_{x}=30 \mu \mathrm{m}, L_{y}=90 \mu \mathrm{m}, L_{c}=20 \mu \mathrm{m}, \\
\eta_{1}=1.34 \times 10^{-3} \mathrm{~Pa} \cdot \mathrm{s}, \eta_{2}=1 \times 10^{-3} \mathrm{~Pa} \cdot \mathrm{s}, \\
\mathrm{Ca}=1 \times 10^{-3}, \theta_{a}=140^{\circ} .
\end{array}\right.
$$

Following experimental results presented in [5], the parameter $L / l_{s}$ in the Cox law (9) is set to $10^{4}$. The evolution of the interface and the global velocity field are shown in Figure 12. The solid line in the fluid zone, corresponds to the interface. The interface is initialized inside the channel. At $t=0.0879$, the contact angle has not exceeded the critical value $\theta_{a}$, thus the triple point is not allowed to move. At $t=0.5977$, the triple point has moved since $\theta_{d}>\theta_{a}$ and the meniscus shows a circular shape.

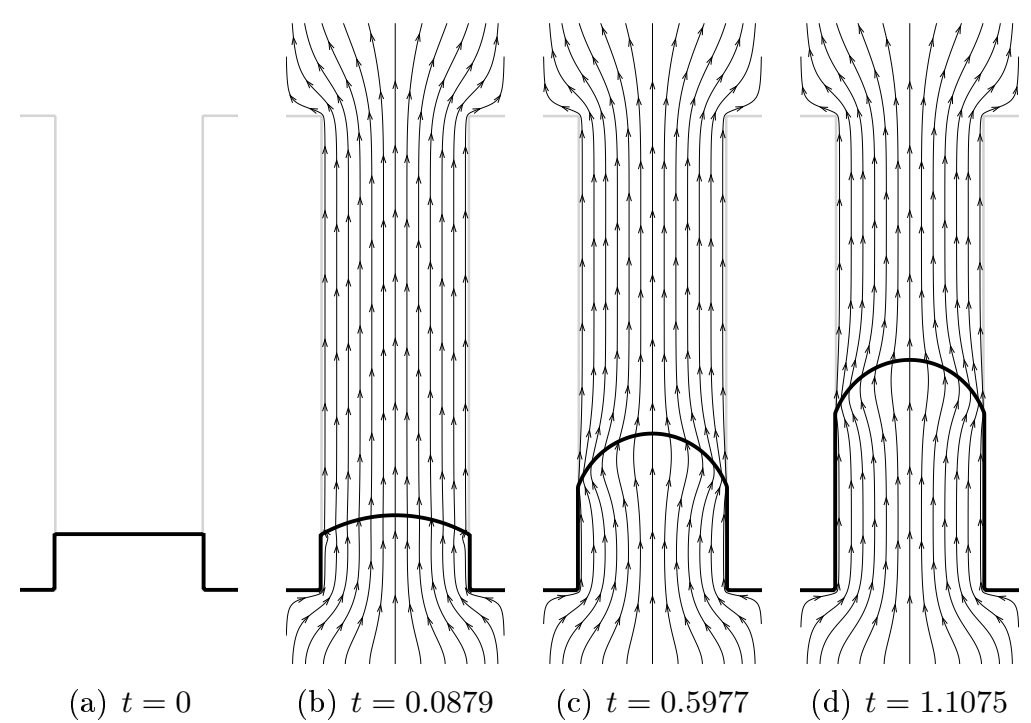

Figure 12: Numerical simulation of the two-phase flow in a channel with two Newtonian fluids. The pictures show the evolution of the interface.

In Figure 13 the relative velocity is shown. The streamlines highlight the recirculation phenomena that are not visible in the global reference. These recirculations show that the relative velocity is tangent to the interface.

The time evolution of the contact angle, is represented in Figure 14. The oscillations of the angle at the beginning of the simulation are caused by the straight initialization of the interface, which generates big velocities near the triple point and therefore some deformations of the interface. At time $t=0.2$, the triple point begins to move according to the increase of the angle $\theta_{d}$, that reaches a stable value around $148^{\circ}$. In Figure 15 a zoom of the flow near the triple point is shown. On the left, the triple point is not allowed to move $\left(\theta_{d}<\theta_{a}\right)$, on the right, $\left(\theta_{d}>\theta_{a}\right)$ the triple point slides along the solid wall with the velocity $V_{c o x}$, which is imposed in the vicinity of the triple point.

Experimentally, it has been demonstrated that for low capillary numbers, the interface has a circular shape [23]. This is confirmed by our simulations as the 


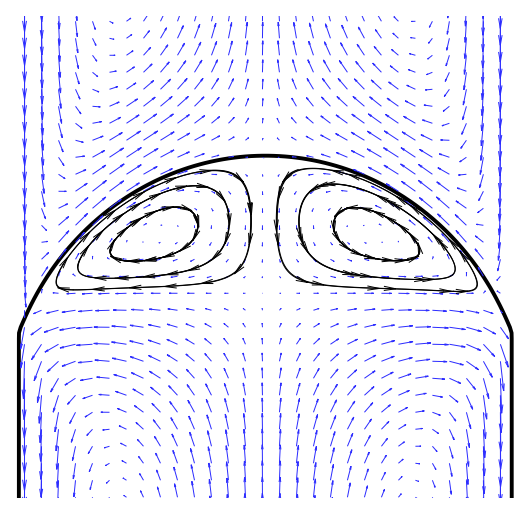

Figure 13: Flow in the vicinity of the interface with the relative velocity vectors. The solid lines are some streamlines.

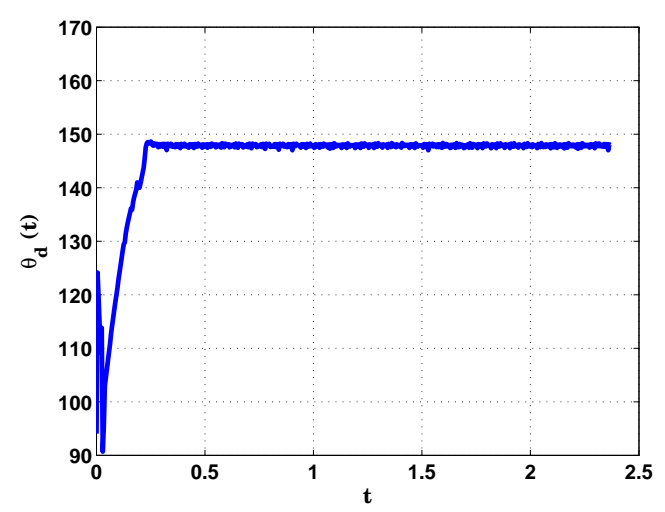

Figure 14: Time evolution of the contact angle of the triple points.

curvature of the interface is constant. Here, the meniscus is superimposed to a circle of radius $R=0.56$ as shown in the Figure 16(a). Besides, the time evolution of the radius reaches very quickly the constant value $R=0.56$ (Figure $16(b))$.

To be confident in the results above, a grid convergence using four grids is performed. The meshes considered are $24 \times 72,48 \times 144,96 \times 288$ and $192 \times 576$. They have been chosen in order to have respectively 15, 30, 60 and 120 cells in the horizontal direction inside the channel. The CPU times for these four cases were $18 \mathrm{~min} 48 \mathrm{~s}, 3 \mathrm{~h} 20 \mathrm{~min}, 35 \mathrm{~h} 18 \mathrm{~min}$ and $374 \mathrm{~h} 24 \mathrm{~min}$. The simulations were carried out using 8 processors on a Dell Precision Workstation T7610 with Intel Xeon CPU E5 - 2630, $2.6 \mathrm{~Hz}, 32 \mathrm{~GB}$ RAM. The results correspond to a dimensionless time equal to 2.01. Unfortunately, the CPU times are quite high as there is a poor parallelism of the computational code. The interface position is presented in the Figure 17(a) and the contact angle evolution is shown in the Figure 17(b). We notice that the interface position obtained by the mesh $24 \times 72$, is very different compared to the position obtained by the other three meshes. In addition, the stable value reached by the contact angle is smaller than the 


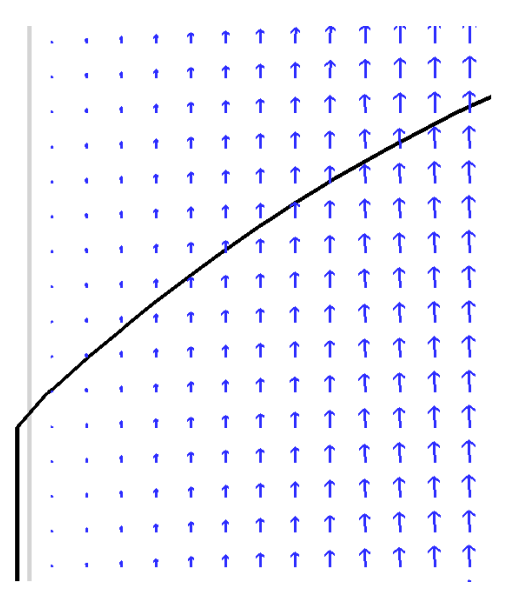

(a) $\theta_{d}<\theta_{a}$

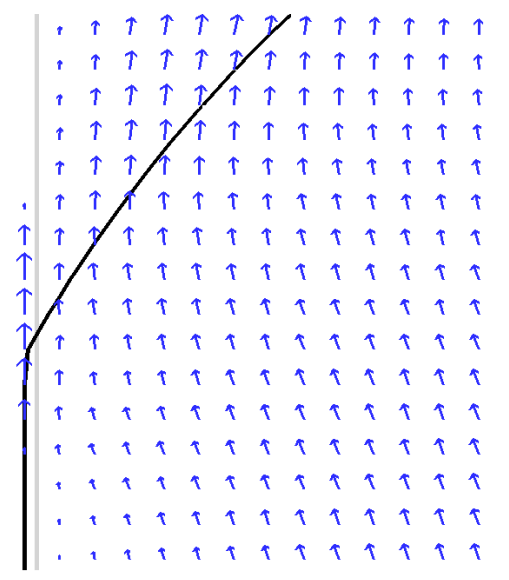

(b) $\theta_{d}>\theta_{a}$

Figure 15: Velocity field near the triple point. (a) The contact angle has not reached its critical value $140^{\circ}$. (b) The contact angle has exceeded its critical value.

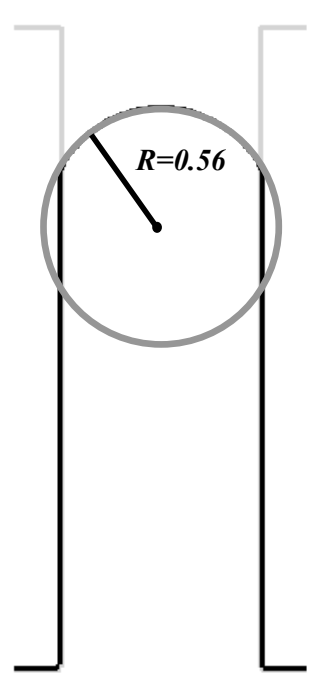

(a)

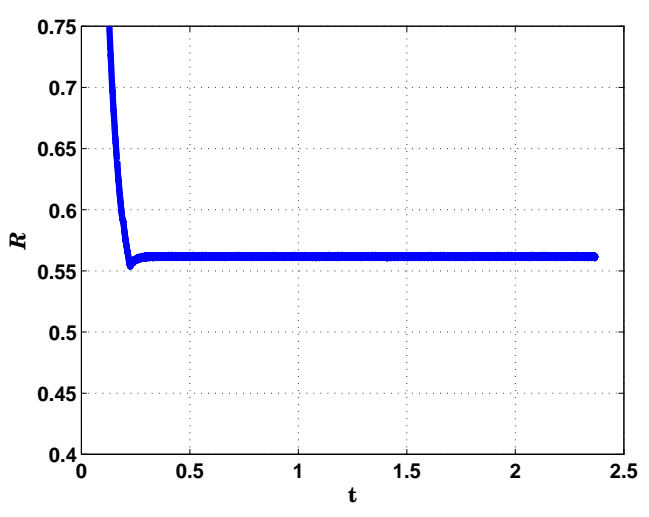

(b)

Figure 16: (a) Comparison between the meniscus shape (black line) and a circle of radius $R=0.56$ (dark grey line). (b) Time evolution of the meniscus radius.

value reached by the other meshes. The results obtained with the grids $96 \times 288$ and $192 \times 576$ are almost identical. So, the grid convergence is reached with 60 cells inside the channel. It can be noticed also that the mesh $48 \times 144$ provides satisfactory results. Thus, to make a good compromise between precision and computational cost, the simulations in a micro-channels network are performed with 30 cells inside each channel.

This convergence test is only valid for the range of capillary numbers we are 


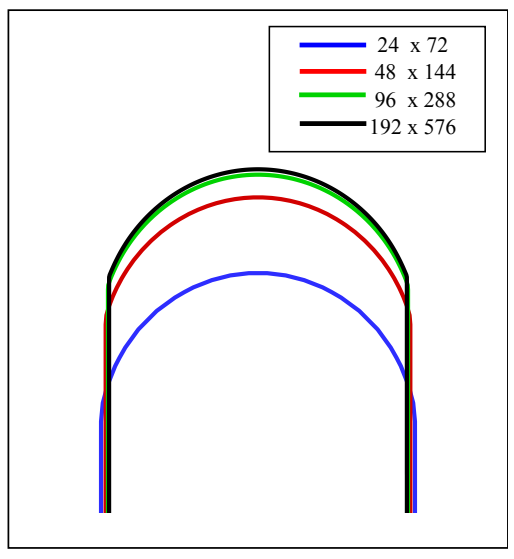

(a)

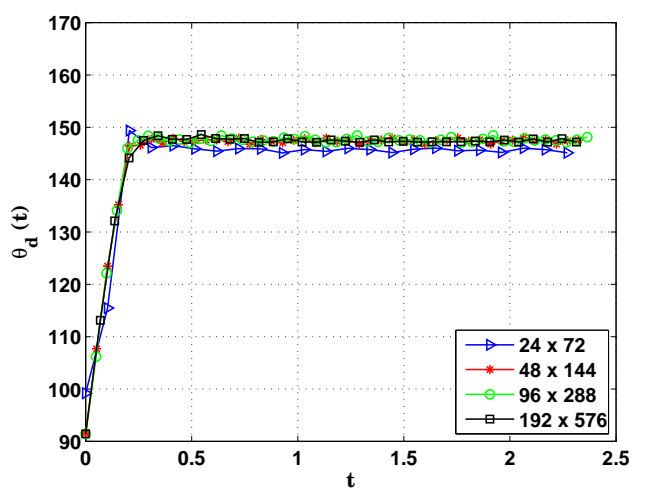

(b)

Figure 17: Grid convergence: (a) position of the interface at $t=2.01$ and (b) contact angle evolution.

interested in, i.e. $C a \in\left[1 \times 10^{-3}, 4 \times 10^{-3}\right]$. For this reason, the convergence test is provided for the smallest $\mathrm{Ca}$ value in this range, which requires a finer mesh than that used for the largest one.

Finally, the last study of this section concerns the meniscus shape with respect to the capillary number and the accuracy of the numerical contact angle method. Six different simulations are performed with $C a \in\left[1 \times 10^{-3}, 4 \times 10^{-3}\right]$ to compare the behavior of the contact angle and the meniscus shape. This is the range of values that is generally used to evaluate the contact angle and the meniscus shape $[5,23,44,46]$.

The mesh is $96 \times 288$ and the physical parameters are:

$$
\left\{\begin{array}{l}
L_{x}=30 \mu \mathrm{m}, L_{y}=90 \mu \mathrm{m}, L_{c}=20 \mu \mathrm{m} \\
\eta_{1}=1.34 \times 10^{-3} \mathrm{~Pa} \cdot s, \eta_{2}=1 \times 10^{-3} \mathrm{~Pa} \cdot s, \\
\theta_{a}=140^{\circ} .
\end{array}\right.
$$

For capillary numbers between $1 \times 10^{-3}$ and $3 \times 10^{-3}$, the meniscus has a circular shape (see Figure 18). We also note that the radius of the meniscus increases when the capillary number decreases. Moreover, when the shape of the meniscus is circular, the radius reaches very quickly its final value (see Figure 19(a)). The time evolution of the contact angle is shown in the Figure 19(b). The qualitative behavior expected is obtained as the contact angle decreases with the capillary number.

We study in Figure 20 the accuracy of the numerical contact angle method. Figure 20 shows the asympthotic values of $\theta_{d}$ as a function of $\ln (\mathrm{Ca})$. The continuous line was obtained by using the Cox law given by equation (9). The relative error is $2 \%$. This error is attributed to the first-order approximation of the penalization term. To improve the accuracy of the penalization method, a second-order approximation of the penalization term could be envisaged [38].

In conclusion, results obtained from the simulations of two Newtonian fluids 


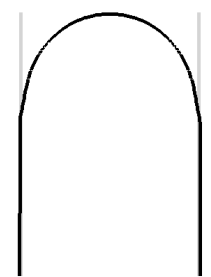

(a) $C a=4 \times 10^{-3}$

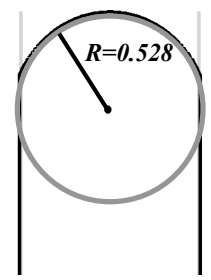

(d) $C a=2 \times 10^{-3}$

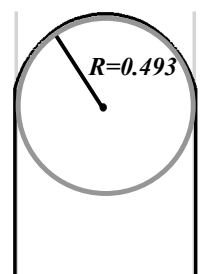

(b) $C a=3 \times 10^{-3}$

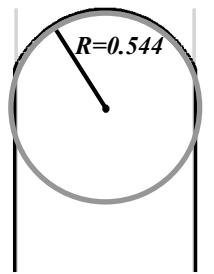

(e) $C a=1.5 \times 10^{-3}$

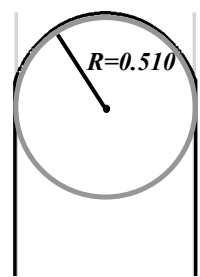

(c) $C a=2.5 \times 10^{-3}$

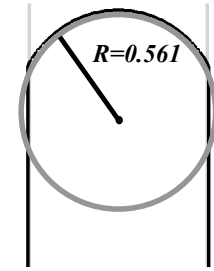

(f) $C a=1 \times 10^{-3}$

Figure 18: Two-phase Newtonian flows with different capillary numbers: meniscus shape at the end of the simulation.

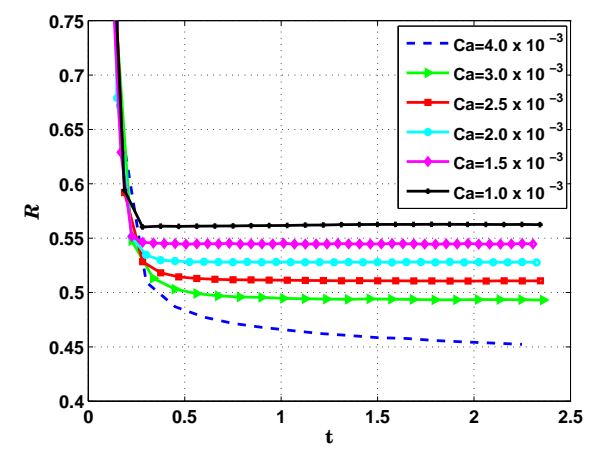

(a)

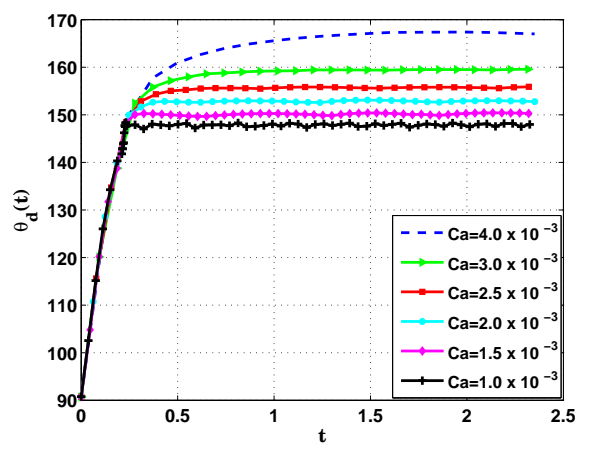

(b)

Figure 19: Two phase-flow of Newtonian fluids with different capillary numbers: (a) time evolution of the meniscus radius and (b) time evolution of the dynamic contact angle.

in a channel are satisfactory from a qualitative point of view: the interface converges very rapidly to a stationary shape. Therefore, the meniscus radius and the dynamic contact angle reach very quickly a stable value. These results agree well with experimental results presented in [23].

\subsection{Newtonian-Viscoelastic case}

We consider the simulation of a two-phase Newtonian-viscoelastic flow in a channel. In this case, a Newtonian fluid is pushed by a viscoelastic one. A grid convergence using three grids is performed. The geometry domain is presented 


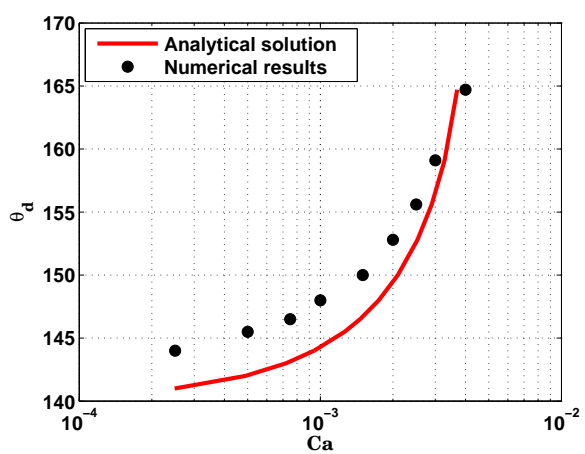

Figure 20: Accuracy of the numerical contact angle method, comparison with the analytical Cox law. The triple line velocity is imposed by first order penalization method.

in the Figure 11 and the physical parameters for simulating the flow are:

$$
\left\{\begin{array}{l}
L_{x}=30 \mu m, L_{y}=90 \mu m, L_{c}=20 \mu m, \\
\eta_{1}=1.34 \times 10^{-3} \mathrm{~Pa} \cdot s, \eta_{2}=1 \times 10^{-3} \mathrm{~Pa} \cdot s, \\
\eta_{p_{2}}=9 \times 10^{-3} \mathrm{~Pa} \cdot s, \lambda_{2}=12 \\
\mathrm{Ca}=1 \times 10^{-3}, \theta_{a}=140^{\circ} .
\end{array}\right.
$$

The meshes considered are $24 \times 72,48 \times 144$ and $96 \times 288$. The CPU times for these three cases were $25 \mathrm{~min} 5 \mathrm{~s}, 4 \mathrm{~h} 7 \mathrm{~min}$ and $52 \mathrm{~h} 19 \mathrm{~min}$. The interface position at dimensionless time $\mathrm{t}=1.5$ is presented in Figure 21 and it shows that we get exactly the same behavior than for the Newtonian-Newtonian case. Thus, to make a good compromise between precision and computational cost, the simulations in a micro-channels network are performed with 30 cells inside each channel.

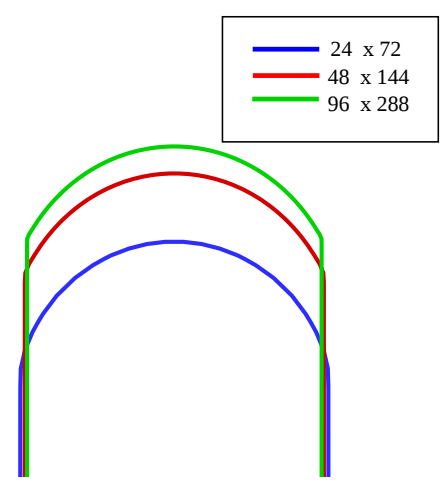

Figure 21: Grid convergence: position of the interface at $t=1.5$ 


\section{Simulations in a micro-channels network}

\subsection{Symmetry test}

In order to validate the detection and the modelization of many triple points in a micro-channels network, the simulation of a two-phase Newtonian flow is performed in a symmetric network. The geometry is shown in the Figure 22. This network is symmetrical with respect to the vertical axis that passes through the middle of the domain and all the channels have the same diameter $L_{c}$. The

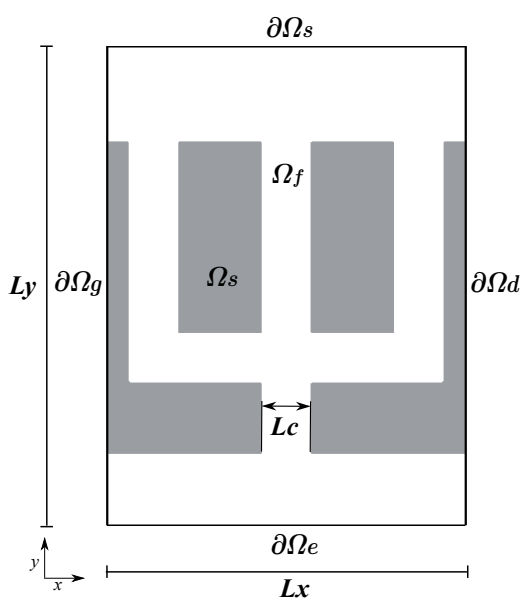

Figure 22: Geometry of the domain for a symmetrical micro-channels network.

physical parameters are:

$$
\left\{\begin{array}{l}
L_{x}=75 \mu \mathrm{m}, L_{y}=100 \mu \mathrm{m}, L_{c}=10 \mu \mathrm{m} \\
\eta_{1}=1.34 \times 10^{-3} \mathrm{~Pa} \cdot \mathrm{s}, \eta_{2}=1 \times 10^{-3} \mathrm{~Pa} \cdot \mathrm{s} \\
\mathrm{Ca}=2.5 \times 10^{-3}, \theta_{a}=140^{\circ} .
\end{array}\right.
$$

The mesh has $270 \times 360$ cells, it has been chosen in order to have at least 30 points in each channel to get relevant qualitative results. Indeed, the computational code should be better parallelized to get lower simulation times and to allow finer grids. The evolution of the interface and the velocity field at different instants are shown in the Figure 23. The interface is initialized in the vertical lower channel. At the beginning of the simulation, two triple points are detected on the left and right walls in this lower channel (see Figure 23(a)). Then, the interface turns on the left and the right channels and two new triple points are detected on the horizontal walls (cf. Figure 23(b)). At instants $t=3.4375$, $t=6.875$ and $t=10.625$ the dynamic of six different triple points must be taken into account in the modelisation of the flow.

In the Figure 23(f) there are ten triple points as some fluid 1 is trapped in the corners. Modeling the triple points near the corners is very special: even if the dynamic angle $\theta_{d}$ is bigger than the critical value $\theta_{a}$, these triple points must remain fix, because the incompressible fluid 1 in the corner does not move. From this simulation, we can verify that the established methodology for the simulation 
of triple points is satisfactory: in a symmetrical micro-channels network, the flow is symmetric and over estimated velocities are not observed near the triple points.

\subsection{Simulation in an heterogeneous network}

Now, we consider the simulation of a two-phase Newtonian-viscoelastic flow in a non symmetrical micro-channels network. The geometry domain is presented in the Figure 24 and the physical parameters are:

$$
\left\{\begin{array}{l}
L_{x}=75 \mu \mathrm{m}, L_{y}=100 \mu \mathrm{m}, L_{c}=10 \mu \mathrm{m}, \\
\eta_{1}=1.34 \times 10^{-3} \mathrm{~Pa} \cdot \mathrm{s}, \eta_{2}=1 \times 10^{-3} \mathrm{~Pa} \cdot \mathrm{s}, \eta_{p_{2}}=9 \times 10^{-3} \mathrm{~Pa} \cdot \mathrm{s}, \\
\mathrm{Ca}=4.5 \times 10^{-3}, \theta_{a}=135^{o} .
\end{array}\right.
$$

The mesh has $270 \times 360$ cells. The evolution of the interface and the streamlines of the flow are shown in the Figure 25. The interface position for three relaxation times $\left(\lambda_{2}=0.1,5.0,12.0\right)$ at two different instants is shown in the Figure 26. We consider that the fluid with $\lambda_{2}=0.1$ is a Newtonian fluid. At $t=4.68$, there are no significant differences with respect to the position of the interface. At $t=16.87$ we notice that the Newtonian fluid enters more easily in the central channel while the fluid with $\lambda_{2}=12$ occupies better the bigger channel. The comparison of the vorticity of relative velocity at the instant $t=14.06$ is presented in the Figure 27 . The most notable differences can be seen in the zone occupied by the viscoelastic fluid. As the relaxation time increases, recirculations in the viscoelastic zone become more important.

From these simulations, it seems that when the relaxation time increases, the viscoelastic fluid tends to better occupy the channels of the network. In order to confirm this result, it is necessary to perform simulations in another geometry and consider fluids with bigger relaxation times than those considered until now. However, numerical instabilities appear when the dimensionless $\lambda_{2}$ relaxation time is bigger than 40. To remedy this problem, a diffusion term is added to the equation of the conformation tensor [47]. The conformation tensor equation from the system of equations (33) becomes:

$$
\frac{\boldsymbol{\tau}-\mathbf{I}}{\lambda(\phi)}+\frac{\partial \boldsymbol{\tau}}{\partial t}+(\mathbf{U} \cdot \nabla) \boldsymbol{\tau}-(\nabla \mathbf{U}) \boldsymbol{\tau}-\boldsymbol{\tau}(\nabla \mathbf{U})^{t}-\boldsymbol{\epsilon} \Delta \boldsymbol{\tau}+\frac{\boldsymbol{\tau}-\boldsymbol{\tau}_{s}}{K}=\mathbf{0},
$$

where $\epsilon$ is a coefficient to be chosen. In this work, we take $\epsilon=10^{-3}$ to restrict the influence of the diffusion term in the equations. Indeed, in [47] the authors have demonstrated that for $\epsilon=10^{-3}$, the diffusion term does not influence the fluid flow. The new geometry is presented in the Figure 28. The parameters of the simulation are:

$$
\left\{\begin{array}{l}
L_{x}=75 \mu \mathrm{m}, L_{y}=100 \mu \mathrm{m}, L_{c}=10 \mu \mathrm{m} \\
\eta_{1}=1.34 \times 10^{-3} \mathrm{~Pa} \cdot \mathrm{s}, \eta_{2}=1 \times 10^{-3} \mathrm{~Pa} \cdot \mathrm{s}, \eta_{p_{2}}=9 \times 10^{-3} \mathrm{~Pa} \cdot \mathrm{s}, \\
\mathrm{Ca}=3 \times 10^{-3}, \theta_{a}=135^{o} .
\end{array}\right.
$$

The mesh has $180 \times 240$ cells and the relaxation times are $\lambda_{2}=0.1$ and $\lambda_{2}=50$. The Figure 29 shows the position of the interface at different instants, for two 
dimensionless relaxation times. At instant $t=7.70$ we can notice that the Newtonian fluid takes advantage in the central channel (see Figure 29(a)) and the viscoelastic fluid goes more easily into the right channel (see Figure 29(b)). This result is confirmed at instant $t=17.50$ where the position of the interface is very different depending on the relaxation time. It can be seen that the viscoelastic fluid tends to better occupy all the network channels (see Figure 29). Because of conservation when the Newtonian fluid goes directly in one direction, it goes less in the others. So the Newtonian fluid goes out quicker than the viscoelastic fluid that better explores the others channels. In the present work no modelisation of the merging of interface lines is performed and so the computation of that case cannot been run a longer time.

\section{Conclusions}

In summary, we have proposed a technique for simulating a two-phase Newtonianviscoelastic flow in a micro-channels network. This technique involves a level-set approach for tracking the interface between the two fluids, a penalization method for dealing with the geometry, a numerical contact angle model based on the Cox's relation and the Oldroyd-B model for modeling the viscoelastic fluid.

The numerical resolution of problems involving a moving interface, requires the implementation of sufficiently high order numerical schemes. For this, a WENO5 scheme is used in the spatial discretization of the transport equation of the level-set function. In addition, to locate the triple points more precisely, this discretization is performed on a twice finer grid in each direction than the mesh used for solving the flow equations.

The first numerical simulations are performed in a two-dimensional straight channel to evaluate the interface shape and the contact angle behavior. At low capillary numbers, the interface converges very quickly to a stationary circular shape. In consequence, the dynamic contact angle also reaches a stable value. Besides, from a qualitative point of view our results are satisfactory as the contact angle decreases with the capillary number.

Then, the proposed methodology for modeling triple points has been evaluated in a symmetrical network. We find that the symmetry of the flow is respected and no over estimated velocities are observed near the triple points. Finally, simulations of two-phase Newtonian-viscoelastic flows are carried out in heterogeneous micro-channels networks. They show that viscoelastic fluids tend to better occupy all the channels of the network as observed in the experiments.

\section{References}

[1] S. Afkhami, S. Zaleski, and M. Bussmann. A mesh-dependent model for applying dynamic contact angles to VOF simulations. J. Comput. Phys., 228(15):5370-5389, 2009. 
[2] P. Angot, C.-H. Bruneau, and P. Fabrie. A penalization method to take into account obstacles in incompressible viscous flows. Numerische Mathematik, 81(4):497-520, 1999.

[3] R. Bird, R. Armstrong, and O. Hassager. Dynamic of polymeric liquids, volume 1. John Wiley \& Sons, 2 edition, 1987.

[4] T. D. Blake. The physics of moving wetting lines. Journal of Colloid and Interface Science, 299(1):1-13, 2006.

[5] D. Bonn, J. Eggers, J. Indekeu, J. Meunier, and E. Rolley. Wetting and spreading. Reviews of Modern Physics, 81(2):739-805, 2009.

[6] C.-H. Bruneau. Boundary conditions on artificial frontiers for incompressible and compressible navier-stokes equations. Mathematical Modelling and Numerical Analysis, 34(2):12, 2000.

[7] B. Bryant. Modeling moving droplets: A precursor film approach. PhD thesis, Harvey Mudd College, Claremont, 2003.

[8] Y. C. Chang, T. Y. Hou, B. Merriman, and S. Osher. A level set formulation of eulerian interface capturing methods for incompressible fluid flows. Journal of Computational Physics, 124(2):449-464, 1996.

[9] H.-Y. Chen, D. Jasnow, and J. Viñals. Interface and contact line motion in a two phase fluid under shear flow. Physical Review Letters, 85(8):1686-1689, 2000 .

[10] D. L. Chopp. Computing minimal surfaces via level set curvature flow. Journal of Computational Physics, 106(1):77-91, 1993.

[11] C. Cottin, H. Bodiguel, and A. Colin. Drainage in two-dimensional porous media: From capillary fingering to viscous flow. Physical Review E, 82(4):046315, 2010.

[12] R. G. Cox. The dynamics of the spreading of liquids on a solid surface. part 1. viscous flow. Journal of Fluid Mechanics, 168:169-194, 1986.

[13] P. G. de Gennes. Wetting statics and dynamics. Reviews of Modern Physics, 57(3):827-863, 1985.

[14] J. A. Diez, L. Kondic, and A. Bertozzi. Global models for moving contact lines. Physical review. E, Statistical, nonlinear, and soft matter physics, 63(1 Pt 1):011208, 2001.

[15] H. Ding and P. D. M. Spelt. Wetting condition in diffuse interface simulations of contact line motion. Physical Review E, 75(4):046708, 2007.

[16] J.-B. Dupont and D. Legendre. Numerical simulation of static and sliding drop with contact angle hysteresis. Journal of Computational Physics, 229(7):2453 - 2478, 2010. 
[17] E. B. Dussan. On the spreading of liquids on solid surfaces: Static and dynamic contact lines. Annual Review of Fluid Mechanics, 11(1):371-400, 1979.

[18] M. Fortin and R. Glowinski. Augmented Lagrangian methods: Applications to the Numerical Solution of Boundary-Value Problems. Elsevier, 1983.

[19] C. Galusinski and P. Vigneaux. On stability condition for bifluid flows with surface tension: Application to microfluidics. Journal of Computational Physics, 227(12):6140-6164, 2008.

[20] P. H. Gaskell, P. K. Jimack, Y.-Y. Koh, and H. M. Thompson. Development and application of a parallel multigrid solver for the simulation of spreading droplets. International Journal for Numerical Methods in Fluids, 56(8):979-989, 2008.

[21] J.-F. Gerbeau and T. Lelievre. Generalized navier boundary condition and geometric conservation law for surface tension. Computer Methods in Applied Mechanics and Engineering, 198(5-8):644-656, 2009.

[22] L. M. Hocking. A moving fluid interface. part 2. the removal of the force singularity by a slip flow. Journal of Fluid Mechanics, 79(02):209-229, 1977.

[23] R. L. Hoffman. A study of the advancing interface. i. interface shape in liquid-gas systems. Journal of Colloid and Interface Science, 50(2):228-241, 1975.

[24] D. Jacqmin. Contact-line dynamics of a diffuse fluid interface. Journal of Fluid Mechanics, 402:57-88, 2000.

[25] G. S. Jiang and C. W. Shu. Efficient implementation of weighted eno schemes. J. Comput. Phys., 126(1):202-228, 1996.

[26] V. V. Khatavkar, P. D. Anderson, and H. E. H. Meijer. Capillary spreading of a droplet in the partially wetting regime using a diffuse-interface model. Journal of Fluid Mechanics, 572:367-387, 2007.

[27] R. G. Larson. Constitutive Equations for Polymer Melts and Solutions. Butterworths, 1988.

[28] E. Lauga, M. Brenner, and H. Stone. Microfluidics: The No-Slip Boundary Condition in Handbook on Experimental Fluid Dynamics. Springer Berlin Heidelberg, 2007.

[29] R. Levi. Partial differentia Equations of Thin Liquid Films: Analysis and Numerical Simulation. PhD thesis, North Carolina State University, Raleigh, 2005.

[30] X. Liu, S. Osher, and T. Chan. Weighted essentially non-oscillatory schemes. Journal of Computational Physics, 115(1):200-212, 1994. 
[31] J. A. Moriarty, L. W. Schwartz, and E. O. Tuck. Unsteady spreading of thin liquid films with small surface tension. Physics of Fluids A: Fluid Dynamics, 3(5):733-742, 1991.

[32] S. J. Osher and R. P. Fedkiw. Level Set Methods and Dynamic Implicit Surfaces. Springer, 1 edition, 2002.

[33] T. Qian, X.-P. Wang, and P. Sheng. Molecular hydrodynamics of the moving contact line in two-phase immiscible flows. Communications in Computational Physics, 1(1):1-52, 2005.

[34] T. Quian. Generalized navier boundary condition for the moving contact line. Communications in Mathematical Sciences, 1(2):333-341, 2003.

[35] E. Rame. Moving contact line problem: state of the contact angle boundary condition. In A. Hubbard, editor, Encyclopedia of Surface and Colloid Science, page 3602-3618. 2002.

[36] W. Ren and W. E. Boundary conditions for the moving contact line problem. Physics of Fluids, 19(2):022101, 2007.

[37] W. J. Rider and D. B. Kothe. Reconstructing volume tracking. Journal of Computational Physics, 141(2):112-152, 1998.

[38] A. Sarthou. Méthodes de domaines fictifs d'ordre élevé pour les équations elliptiques et de Navier Stokes. Application au couplage fluide structure. $\mathrm{PhD}$ thesis, Université Bordeaux 1, Bordeaux, 2009.

[39] R. Scardovelli and S. Zaleski. Direct numerical simulation of free-surface and interfacial flow. Annual Review of Fluid Mechanics, 31(1):567-603, 1999.

[40] L. W. Schwartz and R. R. Eley. Simulation of droplet motion on lowenergy and heterogeneous surfaces. Journal of Colloid and Interface Science, 202(1):173-188, 1998.

[41] P. Seppecher. Moving contact lines in the cahn-hilliard theory. International Journal of Engineering Science, 34(9):977-992, 1996.

[42] J. A. Sethian. A fast marching level set method for monotonically advancing fronts. Proceedings of the National Academy of Sciences of the United States of America, 93(4):1591-1595, 1996.

[43] J. A. Sethian. Level set methods and fast marching methods. Cambridge University Press, 1, 1999.

[44] P. Sheng and M. Zhou. Immiscible-fluid displacement: Contact-line dynamics and the velocity-dependent capillary pressure. Physical Review A, 45(8):5694, 1992.

[45] Y. D. Shikhmurzaev. Capillary Flows with Forming Interfaces. Chapman and Hall/CRC, 2007. 
[46] P. D. Spelt. A level-set approach for simulations of flows with multiple moving contact lines with hysteresis. Journal of Computational Physics, 207(2):389-404, 2005.

[47] R. Sureshkumar and A. N. Beris. Effect of artificial stress diffusivity on the stability of numerical calculations and the flow dynamics of time-dependent viscoelastic flows. Journal of Non-Newtonian Fluid Mechanics, 60(1):53 80,1995 .

[48] M. Sussman. A method for overcoming the surface tension time step constraint in multiphase flows ii. International Journal for Numerical Methods in Fluids, 68(11):1343-1361, 2012.

[49] S. Tancogne. Calcul numérique et stabilité d écoulements diphasiques tridimiensionnels en microfluidique. PhD thesis, Université Bordeaux 1, Bordeaux, 2007.

[50] P. A. Thompson and M. O. Robbins. Simulations of contact-line motion: Slip and the dynamic contact angle. Physical Review Letters, 63(7):766-769, 1989.

[51] S. O. Unverdi and G. Tryggvason. A front tracking method for viscous, incompressible, multi-fluid flows. Journal of Computational Physics, 100(1):25-37, 1992.

[52] P. Vigneaux. Méthodes Level Set pour des problèmes d'interface en microfluidique. PhD thesis, Université Bordeaux 1, Bordeaux, 2007.

[53] C. Walker and B. Müller. Contact line treatment with the sharp interface method. Computers \&3 Fluids, 84(0):255 - 261, 2013.

[54] R. Wang and R. J. Spiteri. Linear instability of the fifth-order weno method. SIAM Journal on Numerical Analysis, 45(5):1871-1901, 2007.

[55] Y. L. Xiong. Analyse par simulation numérique de la réduction de la traînée et des caractéristiques d'écoulements bidimensionnels par l'ajout de polymères en solution. PhD thesis, Université Bordeaux 1, Bordeaux, 2010.

[56] P. Yue and J. J. Feng. Phase-field simulations of dynamic wetting of viscoelastic fluids. Journal of Non-Newtonian Fluid Mechanics, 189-190(0):8 $-13,2012$.

[57] S. Zahedi, K. Gustavsson, and G. Kreiss. A conservative level set method for contact line dynamics. Journal of Computational Physics, 228(17):6361-6375, 2009. 


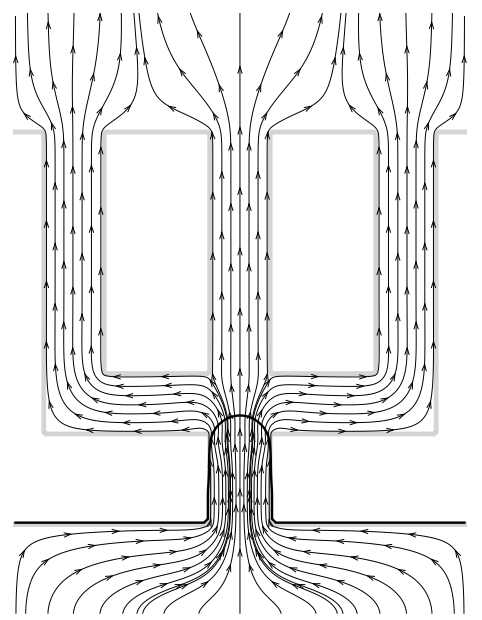

(a) $t=1.25$

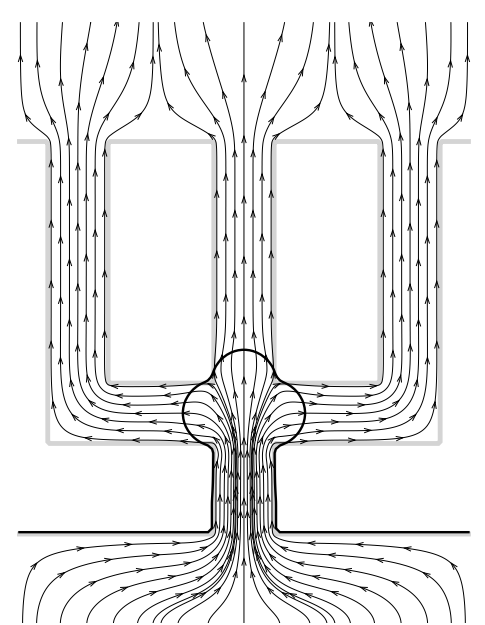

(c) $t=3.4375$

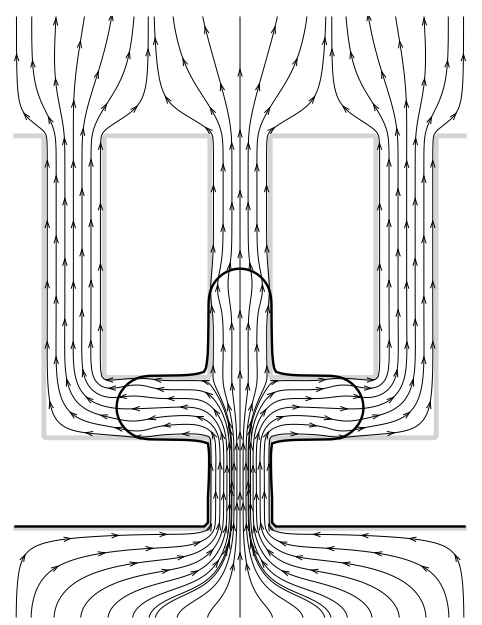

(e) $t=10.625$

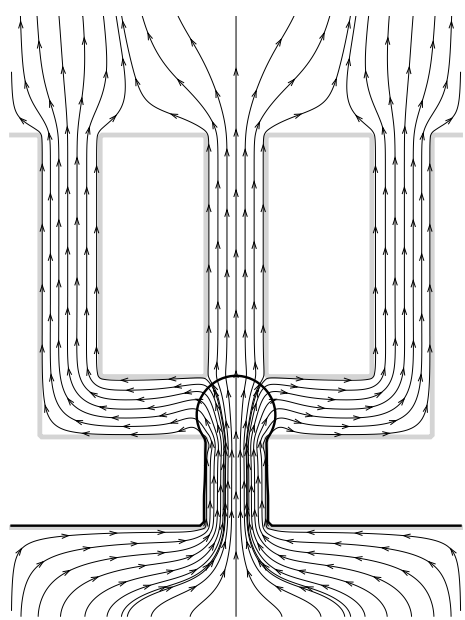

(b) $t=2.1875$

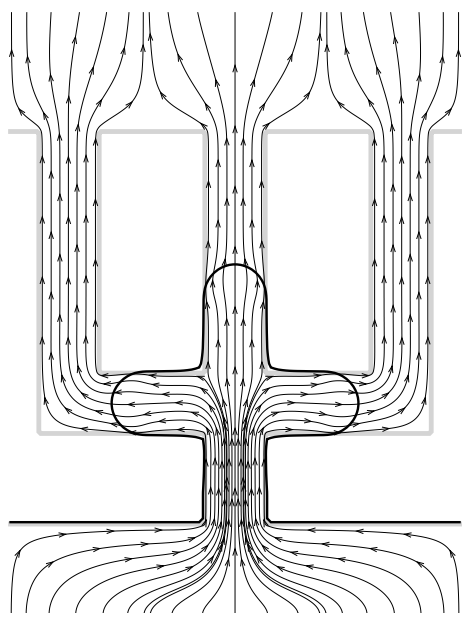

(d) $t=6.875$

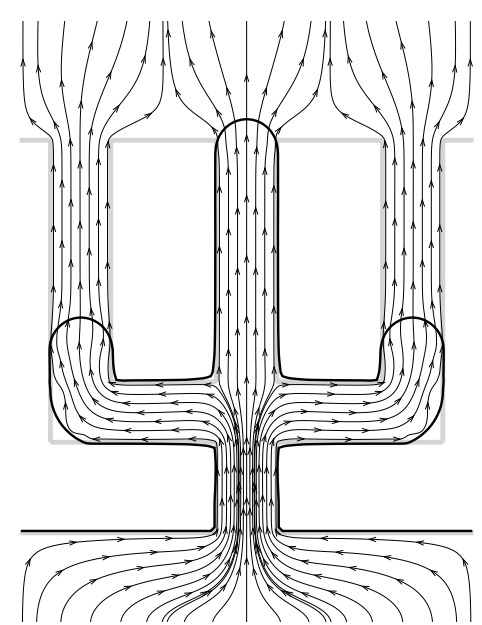

(f) $t=14.062$

Figure 23: Simulation of a two-phase Newtonian flow in a symmetrical network. Evolution of the interface and velocity field at different instants. 


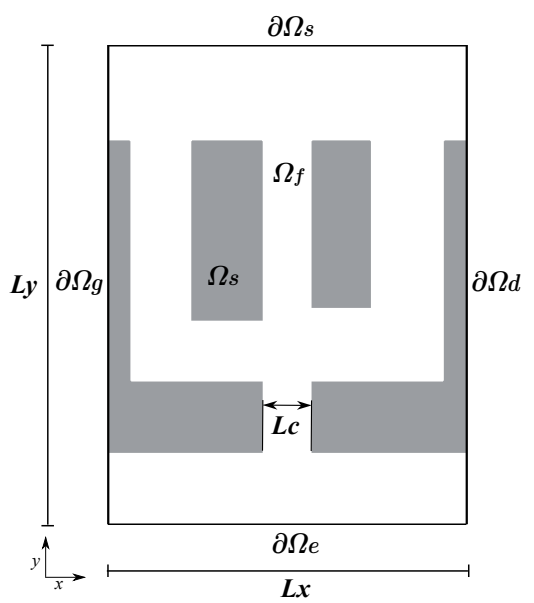

Figure 24: Geometry of the domain for a non symmetrical network. 


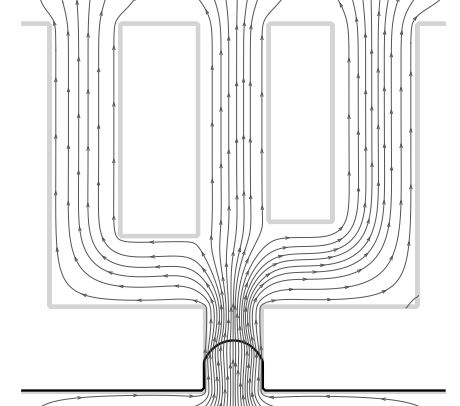

(a) $t=0.3$

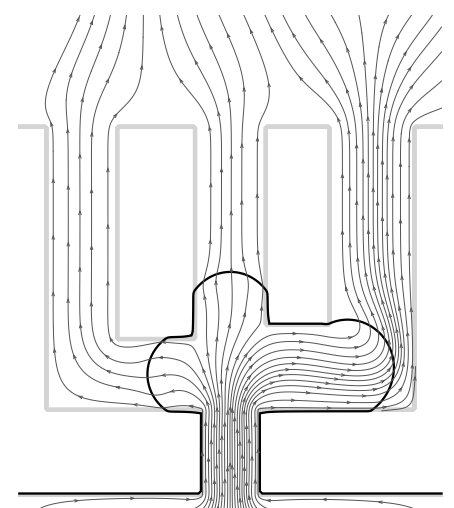

(c) $t=8.2$

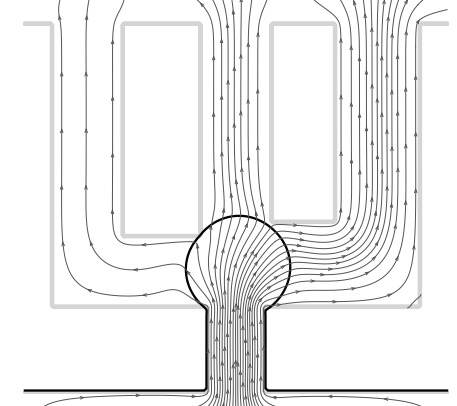

(b) $t=3.5$

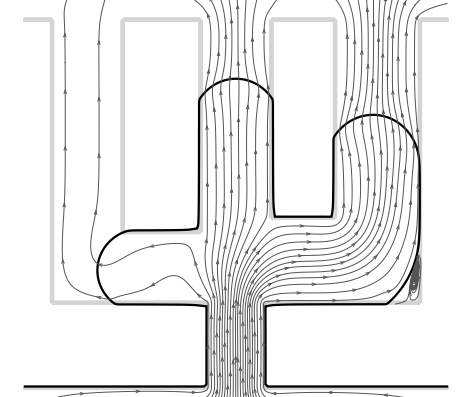

(d) $t=14.4$

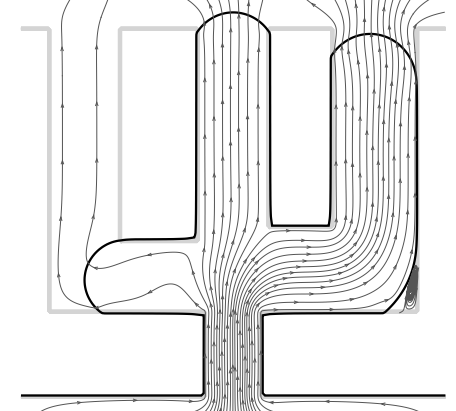

(e) $t=18.75$

Figure 25: Two-phase Newtonian-viscoelastic flow: streamlines and interface evolution. 


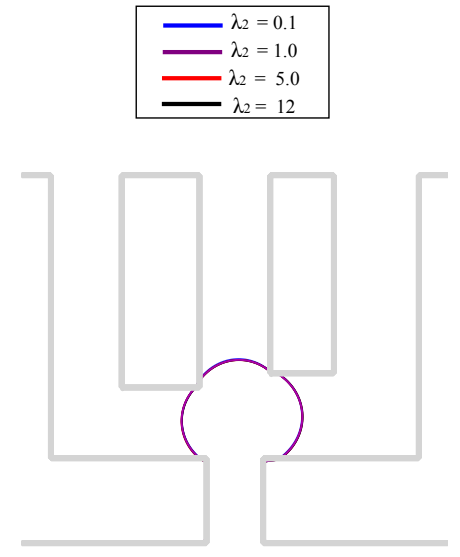

(a) $\mathrm{t}=4.68$

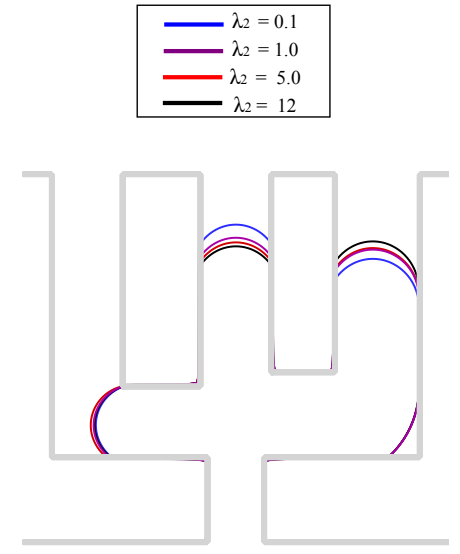

(b) $t=16.87$

Figure 26: Comparison of the interface position for different relaxation times.

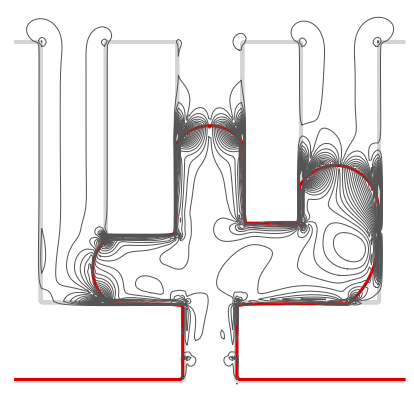

(a) $\lambda_{2}=0.1$

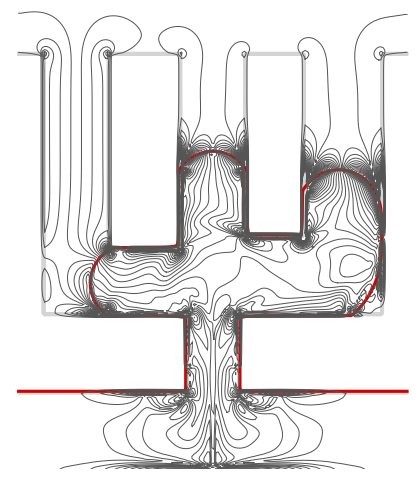

(c) $\lambda_{2}=5.0$

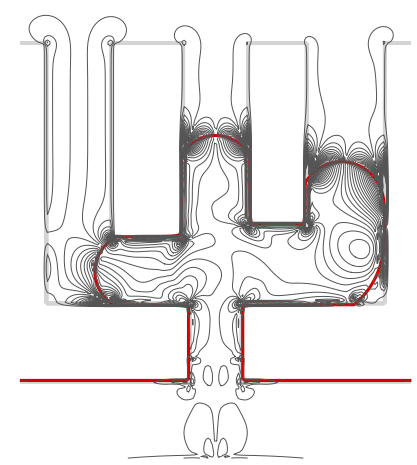

(b) $\lambda_{2}=1.0$

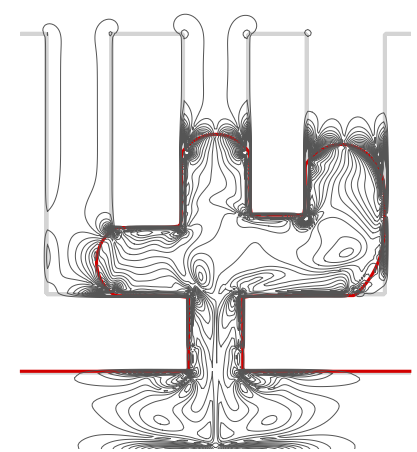

(d) $\lambda_{2}=12$

Figure 27: Vorticity of the relative velocity at $t=14.06$. 


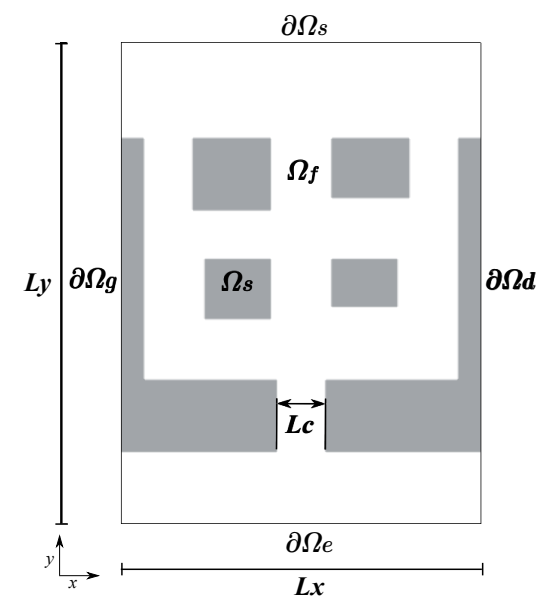

Figure 28: Geometry of the domain for a non symmetrical network with connexions between the channels.

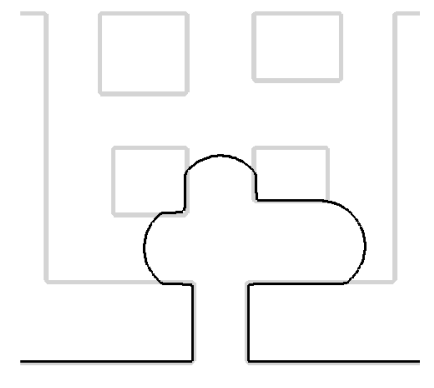

(a) $\lambda_{2}=0.1$

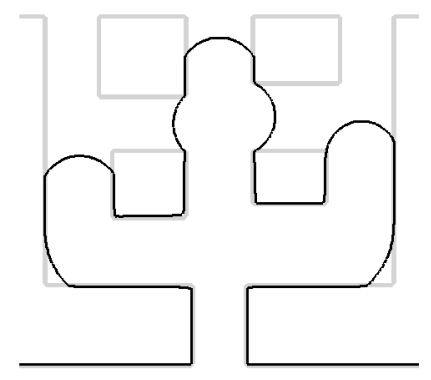

(c) $\lambda_{2}=0.1$

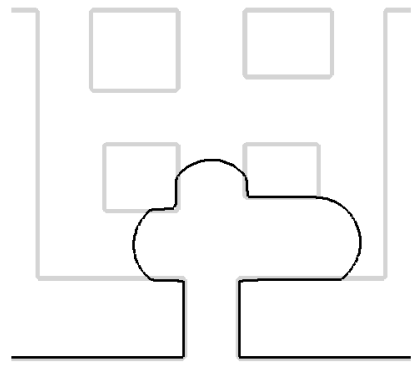

(b) $\lambda_{2}=50$

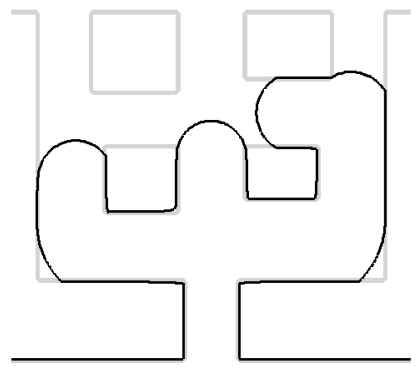

(d) $\lambda_{2}=50$

Figure 29: Comparison of the interface position at instant $t=7.70$ (top) and $t=17.50$ (bottom). 